\title{
Generalized kinetic equations for charge carriers in graphene
}

\author{
M. Auslender ${ }^{1}$ and M. I. Katsnelson ${ }^{2}$ \\ ${ }^{1}$ Ben-Gurion University of the Negev, \\ POB 653, Beer Sheva 84105, Israel \\ ${ }^{2}$ Institute for Molecules and Materials, Radboud University of Nijmegen, \\ NL-6525 ED Nijmegen, The Netherlands
}

(Dated: October 24, 2018)

\begin{abstract}
A system of generalized kinetic equations for the distribution functions of two-dimensional Dirac fermions scattered by impurities is derived in the Born approximation with respect to short-range impurity potential. It is proven that the conductivity following from classical Boltzmann equation picture, where electrons or holes have scattering amplitude reduced due chirality, is justified except for an exponentially narrow range of chemical potential near the conical point. When in this range, creation of infinite number of electron-hole pairs related to quasi-relativistic nature of electrons in graphene results in a renormalization of minimal conductivity as compared to the Boltzmann term and logarithmic corrections in the conductivity similar to the Kondo effect.
\end{abstract}




\section{Introduction}

Recent discovery of two-dimensional (2D) allotrope of carbon, graphene, and experimental demonstration of its massless Dirac energy spectrum has initiated a huge experimental and theoretical activity in the field (for review, see Refs, 1, 2, 3). One of the most interesting aspects of the graphene physics from theoretical point of view is a deep and fruitful relation with the quantum electrodynamics. $\underline{4}, 5,6,7,8,9,10$ In particular, anomalous transport properties of 2D Dirac fermions, such as finite conductivity of order of $e^{2} / h$ in the limit of zero charge carrier concentration $11,12,13,14,15,16$ can be associated with a specific quantum relativistic phenomenon known as Zitterbewegung. $\stackrel{7}{-}$ The current operator of non-relativistic electron commutes with its kinetic-energy Hamiltonian and does not commute with the potential-energy one. Yet, it is vice versa for the Dirac electrons that is a reason for the Zitterbewegung. The same commutation properties hold for graphene in the case where the potential does not cause Umklapp process. Qualitatively, an impurity potential acting on non-relativistic electron creates random friction-like force which causes finite conductivity. This is expressed quantitatively in the standard theory of electronic transport in disordered metals and semiconductors $17, \underline{18,19,20}$ by deriving and solving the classical Boltzmann equation. The impurity potential action on the Dirac electron can not be described within such a simple picture. Despite this important difference many authors exploited the classical Boltzmann equation to analyze electron transport in graphene $10,16,21,22,23,24$ Rigorously speaking, it is not clear what will be the limits of its applicability in this unusual situation. Our work presents a consequent derivation of kinetic equations for the 2D massless Dirac fermions. Some of our results for the static conductivity are similar to those obtained by various quantum-field theory methods. $11,12,13,14,15,16$ The approach based on the kinetic equation provides an alternative view on the anomalous transport properties of graphene. It can be easier generalized for more complicated situations such as strong electric fields, hot electrons, etc. These issues are beyond the scope of the present work. We will not consider also the effects of Anderson localization and antilocalization $25,26,27,28$ in graphene restricting ourselves by the case of a weak disorder in the leading-order approximation. As we will see even in this case the problem turns out to be very nontrivial and instructive. We will prove that for not too small doping the standard Boltzmann equation with the scattering amplitude specific for massless fermions does give the leading term in the conductivity and 
will find corrections to it due to the Zitterbewegung. In particular, these corrections have an interesting temperature dependence similar to the Kondo effect.

A general idea of the approach used here is traced back to seminal papers by Kohn and Luttinger. ${ }^{29}$ Starting from Schrödinger equation for noninteracting electrons in a random impurity potential they consequently derived the kinetic equation for diagonal (in momentum representation) matrix elements of the one-electron density matrix in the cases of weak potential or small impurity concentration. In these cases the kinetic equation turned out to be identical with the classical Boltzmann equation. Even for the simplest system to which it was initially applied, the Kohn and Luttinger treatment ${ }^{29}$ proved not simple. For multicomponent systems one may also follow the route of Ref.29 and infer on existence of a closed system for distribution functions in the momentum space - usual ones and functions that describe inter-subsystems transitions - but complexity of deriving such kinetic equations sharply increases.

Several established formalisms exist nowadays, which automate the above derivation assuming existence of some kinetic equations in principle. A partial list includes KadanoffBaym $^{30}$, Keldysh $\underline{31}, \underline{32}$, Zubarev nonequilibrium statistical operator (NSO) ${ }^{33}$ (for the NSO method, see also recent reviews $\left.{ }^{34}, 35\right)$ and Peletminskii-Yatsenko ${ }^{36}$ methods. The Keldysh, NSO and Peletminskii-Yatsenko methods have close rationales. Namely, existence of an asymptotic density matrix which allows for Wick-rule decoupling of the creation and annihilation operators product averages is assumed in these methods. The consideration of non-equilibrium at strong interactions benefits using the Keldysh method which is distinguished for highly developed diagram technique. At weak interactions, however, when the Born approximation is applicable the simplest approach in our opinion is with the NSO and Peletminskii-Yatsenko methods. This is because in the Born approximation, closed equations for the averages of gross variables, generalized kinetic equations (GKE), which describe non-equilibrium of interest (provided that such variables are declared in advance) were derived within these frameworks in late 60's once for all. $33,34,35,36$

In this paper we obtain and asymptotically solve GKE for spatially homogeneous graphene in order to calculate the linear-response conductivity. The main difference with the canonical $\operatorname{case}^{29}$ is that for graphene the diagonal in the momentum representation average density matrix is still two by two matrix in the pseudospin space, its off-diagonal elements describing the Zitterbewegung. This makes the GKE structure, on the whole, es- 
sentially different from that of the classical kinetic equation. The structure of the paper is the following. In section I we present original expressions for the Hamiltonian, current and coordinate operators. In section II we specify the gross variables appropriate for the kinetics in spatially homogeneous case, which in fact are all the density matrix elements, and on their base concretize GKE regarding the interaction with arbitrary static impurities as a perturbation. In section III, assuming presence of a thermostat, we consider linear response regime (the case of small electric field) and express the linear static conductivity via two unknown functions of the one-electron energy. These functions, together with a subsidiary function of energy, satisfy a coupled system of linear integral equations resulting from linearizing GKE in electric field strength. In section IV, we solve the linearized system and calculate the conductivity within an ultraviolet cut off Dirac-delta impurity potential asymptotically in a controllable small parameter, using methods of solving singular integral equations. In section $\mathrm{V}$ we discuss the results obtained.

\section{PRELIMINARIES}

We proceed with the Hamiltonian of two-dimensional massless Dirac fermions describing charge carriers in graphene if one neglects the Umklapp processes between valleys $K$ and $K^{\prime}$

$$
\mathcal{H}_{0}=v \sum_{\mathbf{p}} \Psi_{\mathbf{p}}^{\dagger}(\boldsymbol{\tau} \cdot \mathbf{p}) \Psi_{\mathbf{p}}
$$

where $\mathbf{p}$ is the momentum vector, $v$ is the velocity,

$$
\Psi_{\mathbf{p}}=\left(\begin{array}{c}
\psi_{\mathbf{p} 1} \\
\psi_{\mathbf{p} 2}
\end{array}\right), \Psi_{\mathbf{p}}^{\dagger}=\left(\begin{array}{ll}
\psi_{\mathbf{p} 1}^{\dagger} & \psi_{\mathbf{p} 2}^{\dagger}
\end{array}\right)
$$

are two-component pseudospinor operators, 1,2 labelling the sublattices, and

$$
\boldsymbol{\tau}=\left(\tau_{x}, \tau_{y}\right), \tau_{x}=\left(\begin{array}{ll}
0 & 1 \\
1 & 0
\end{array}\right), \tau_{y}=\left(\begin{array}{cc}
0 & -i \\
i & 0
\end{array}\right)
$$

are the Pauli matrices in the pseudospin space. We will neglect here real spin and valley indices. The Hamiltonian (1) can be diagonalized using the unitary transformation matrix 16

$$
U_{\mathbf{p}}=\frac{1}{\sqrt{2}}\left(\begin{array}{cc}
1 & 1 \\
e^{i \phi_{\mathbf{p}}} & -e^{i \phi_{\mathbf{p}}}
\end{array}\right)
$$


where $\phi_{\mathbf{p}}$ is the polar angle of the vector $\mathbf{p}$. Hence the new electron operators given by

$$
\Xi_{\mathbf{p}}=U_{\mathbf{p}}^{\dagger} \Psi_{\mathbf{p}}=\frac{1}{\sqrt{2}}\left(\begin{array}{c}
\psi_{\mathbf{p} 1}+e^{-i \phi_{\mathbf{p}}} \psi_{\mathbf{p} 2} \\
\psi_{\mathbf{p} 1}-e^{-i \phi_{\mathbf{p}}} \psi_{\mathbf{p} 2}
\end{array}\right)=\left(\begin{array}{c}
\xi_{\mathbf{p} 1} \\
\xi_{\mathbf{p} 2}
\end{array}\right)
$$

and

$$
\Xi_{\mathbf{p}}^{\dagger}=\Psi_{\mathbf{p}}^{\dagger} U_{\mathbf{p}}=\left(\begin{array}{ll}
\xi_{\mathbf{p} 1}^{\dagger} & \xi_{\mathbf{p} 2}^{\dagger}
\end{array}\right)
$$

are the annihilation and creation operators of the conduction and valence band electrons. Thus we have

$$
\Psi_{\mathbf{p}}=U_{\mathbf{p}} \Xi_{\mathbf{p}}, \Psi_{\mathbf{p}}^{\dagger}=\Xi_{\mathbf{p}}^{\dagger} U_{\mathbf{p}}^{\dagger}
$$

and

$$
\mathcal{H}_{0}=v \sum_{\mathbf{p}, s= \pm 1} s p \xi_{\mathbf{p} s}^{\dagger} \xi_{\mathbf{p} s} .
$$

In what follows we will consider the simplest case where electrons experience action of a scalar potential $V(\mathbf{r})$ presents. The interaction Hamiltonian in this case is given by

$$
\mathcal{H}_{\mathrm{int}}=S^{-1} \sum_{\mathbf{p p}^{\prime}} V\left(\mathbf{p}-\mathbf{p}^{\prime}\right) \Psi_{\mathbf{p}}^{\dagger} \Psi_{\mathbf{p}^{\prime}}=S^{-1} \sum_{\mathbf{p p}^{\prime}} \Xi_{\mathbf{p}}^{\dagger} \widehat{V}_{\mathbf{p p}^{\prime}} \Xi_{\mathbf{p}^{\prime}}
$$

where $S$ is the graphene layer surface area, $V(\mathbf{q})$ is the Fourier transform of $V(\mathbf{r})$ and

$$
\widehat{V}_{\mathbf{p p}^{\prime}}=\frac{1}{2} V\left(\mathbf{p}-\mathbf{p}^{\prime}\right)\left(\begin{array}{cc}
1+e^{-i\left(\phi_{\mathbf{p}}-\phi_{\mathbf{p}^{\prime}}\right)} & 1-e^{-i\left(\phi_{\mathbf{p}}-\phi_{\mathbf{p}^{\prime}}\right)} \\
1-e^{-i\left(\phi_{\mathbf{p}}-\phi_{\mathbf{p}^{\prime}}\right)} & 1+e^{-i\left(\phi_{\mathbf{p}^{\prime}-\phi_{\mathbf{p}^{\prime}}}\right)}
\end{array}\right) .
$$

The current density operator in the new variables reads

$$
\mathbf{J}=e v \sum_{\mathbf{p}} \Psi_{\mathbf{p}}^{\dagger} \tau \Psi_{\mathbf{p}}=\sum_{\mathbf{p}} \Xi_{\mathbf{p}}^{\dagger} \mathbf{j}_{\mathbf{p}} \Xi_{\mathbf{p}}
$$

where

$$
\mathbf{j}_{\mathbf{p}}=e v U_{\mathbf{p}}^{\dagger} \boldsymbol{\tau} U_{\mathbf{p}}=\left(j_{\mathbf{p} x}, j_{\mathbf{p} y}\right)
$$

and

$$
j_{\mathbf{p} x}=e v\left(\begin{array}{cc}
\cos \phi_{\mathbf{p}} & -i \sin \phi_{\mathbf{p}} \\
i \sin \phi_{\mathbf{p}} & -\cos \phi_{\mathbf{p}}
\end{array}\right), j_{\mathbf{p} y}=e v\left(\begin{array}{cc}
\sin \phi_{\mathbf{p}} & i \cos \phi_{\mathbf{p}} \\
-i \cos \phi_{\mathbf{p}} & -\sin \phi_{\mathbf{p}}
\end{array}\right) \text {. }
$$


Off-diagonal elements of the current operator correspond to the Zitterbewegung processes $\underline{\underline{T}}^{\underline{T}}$ For the $x$ and $y$-components of the current, we further obtain

$$
\begin{aligned}
& J_{x}=e v \sum_{\mathbf{p}} \Xi_{\mathbf{p}}^{\dagger}\left(\begin{array}{cc}
\cos \phi_{\mathbf{p}} & -i \sin \phi_{\mathbf{p}} \\
i \sin \phi_{\mathbf{p}} & -\cos \phi_{\mathbf{p}}
\end{array}\right) \Xi_{\mathbf{p}} \\
& =e v \sum_{\mathbf{p}}\left[\cos \phi_{\mathbf{p}}\left(\xi_{\mathbf{p}, 1}^{\dagger} \xi_{\mathbf{p}, 1}-\xi_{\mathbf{p},-1}^{\dagger} \xi_{\mathbf{p},-1}\right)-i \sin \phi_{\mathbf{p}}\left(\xi_{\mathbf{p}, 1}^{\dagger} \xi_{\mathbf{p},-1}-\xi_{\mathbf{p},-1}^{\dagger} \xi_{\mathbf{p}, 1}\right)\right],
\end{aligned}
$$

and

$$
\begin{aligned}
J_{y} & =e v \sum_{\mathbf{p}} \Xi_{\mathbf{p}}^{\dagger}\left(\begin{array}{cc}
\sin \phi_{\mathbf{p}} & i \cos \phi_{\mathbf{p}} \\
-i \cos \phi_{\mathbf{p}} & -\sin \phi_{\mathbf{p}}
\end{array}\right) \Xi_{\mathbf{p}} \\
& =e v \sum_{\mathbf{p}}\left[\sin \phi_{\mathbf{p}}\left(\xi_{\mathbf{p}, 1}^{\dagger} \xi_{\mathbf{p}, 1}-\xi_{\mathbf{p},-1}^{\dagger} \xi_{\mathbf{p},-1}\right)+i \cos \phi_{\mathbf{p}}\left(\xi_{\mathbf{p}, 1}^{\dagger} \xi_{\mathbf{p},-1}-\xi_{\mathbf{p},-1}^{\dagger} \xi_{\mathbf{p}, 1}\right)\right] .
\end{aligned}
$$

At last, the electron coordinate operator which is necessary to derive the field term in the kinetic equation reads

$$
\mathbf{R}=i \sum_{\mathbf{p}} \Psi_{\mathbf{p}}^{\dagger} \nabla \Psi_{\mathbf{p}}
$$

where $\nabla$ is the gradient operator with respect to the momentum p. Using the above unitary transformation, we get

$$
\begin{aligned}
\mathbf{R} & =i \sum_{\mathbf{p}} \Xi_{\mathbf{p}}^{\dagger} U_{\mathbf{p}}^{\dagger} \boldsymbol{\nabla}\left(U_{\mathbf{p}} \Xi_{\mathbf{p}}\right)=i \sum_{\mathbf{p}} \Xi_{\mathbf{p}}^{\dagger} \boldsymbol{\nabla} \Xi_{\mathbf{p}}+i \sum_{\mathbf{p}} \Xi_{\mathbf{p}}^{\dagger} U_{\mathbf{p}}^{\dagger}\left[\boldsymbol{\nabla} \frac{1}{\sqrt{2}}\left(\begin{array}{cc}
1 & 1 \\
e^{i \phi_{\mathbf{p}}}-e^{i \phi_{\mathbf{p}}}
\end{array}\right) U_{\mathbf{p}}\right] \Xi_{\mathbf{p}} \\
& =\sum_{\mathbf{p}}\left[i\left(\xi_{\mathbf{p} 1}^{\dagger} \boldsymbol{\nabla} \xi_{\mathbf{p} 1}+\xi_{\mathbf{p},-1}^{\dagger} \boldsymbol{\nabla} \xi_{\mathbf{p},-1}\right)-\frac{1}{2}\left(\xi_{\mathbf{p} 1}^{\dagger} \xi_{\mathbf{p} 1}+\xi_{\mathbf{p},-1}^{\dagger} \xi_{\mathbf{p},-1}\right) \nabla \phi_{\mathbf{p}}\right] \\
& +\frac{1}{2} \sum_{\mathbf{p}} \boldsymbol{\nabla} \phi_{\mathbf{p}}\left(\xi_{\mathbf{p} 1}^{\dagger} \xi_{\mathbf{p},-1}+\xi_{\mathbf{p},-1}^{\dagger} \xi_{\mathbf{p} 1}\right) .
\end{aligned}
$$

To simplify $\mathbf{R}$ let us perform additional gauge transformation $\xi_{\mathbf{p} s} \rightarrow e^{-i \frac{1}{2} \phi_{\mathbf{p}}} \xi_{\mathbf{p} s}$ that retains the Hamiltonian $\mathcal{H}_{0}$ unchanged but renormalizes the coordinate and current density operators to take the form

$$
\mathbf{R} \rightarrow \sum_{\mathbf{p}, m= \pm 1}\left(i \xi_{\mathbf{p} m}^{\dagger} \nabla \xi_{\mathbf{p} m}+\frac{1}{2} \xi_{\mathbf{p} m}^{\dagger} \xi_{\mathbf{p},-m} \nabla \phi_{\mathbf{p}}\right)=\mathbf{R}_{\text {intra }}+\mathbf{R}_{\text {inter }}
$$

and

$$
\mathbf{J} \rightarrow e v \sum_{\mathbf{p}, m= \pm 1} m\left(\frac{\mathbf{p}}{p} \xi_{\mathbf{p} m}^{\dagger} \xi_{\mathbf{p} m}+i \xi_{\mathbf{p} m}^{\dagger} \xi_{\mathbf{p},-m} p \nabla \phi_{\mathbf{p}}\right)=\mathbf{J}_{\text {intra }}+\mathbf{J}_{\text {inter }}
$$


respectively. Here we have separated explicitly intraband (electron-electron and hole-hole) and interband (electron-hole) contributions. Note that

$$
\frac{d}{d t} e \mathbf{R}=\mathbf{J}
$$

as it should be. The interaction matrix elements are thus transformed to

$$
\widehat{V}_{\mathbf{p p}^{\prime}} \rightarrow V\left(\mathbf{p}-\mathbf{p}^{\prime}\right)\left(\begin{array}{cc}
\cos \frac{\phi_{\mathbf{p}}-\phi_{\mathbf{p}^{\prime}}}{2} & i \sin \frac{\phi_{\mathbf{p}}-\phi_{\mathbf{p}^{\prime}}}{2} \\
i \sin \frac{\phi_{\mathbf{p}}-\phi_{\mathbf{p}^{\prime}}}{2} & \cos \frac{\phi_{\mathbf{p}}-\phi_{\mathbf{p}^{\prime}}}{2}
\end{array}\right)
$$

\section{THE BORN-APPROXIMATION KINETIC EQUATIONS}

\section{A. General outline}

The basic idea of the methods of Refs,33,36 is a concept of so called "coarse-grained" dynamics. To apply the formalism we are, as noted in Introduction, to suggest the gross variables $P$, averages of which $\langle P\rangle$ at the kinetic stage of the evolution are believed to satisfy GKE. It was proven by Kohn and Luttinger 29 that, if $V(\mathbf{r})$ is due to random impurities, the diagonal elements of the one-electron density matrix in the momentum representation averaged over weakly perturbed non-equilibrium ensemble are self averaging over the impurity configurations and do obey such a reduced description, at least for weak enough potential or small impurity concentration. Our problem is formally different from standard one only in existence of the interband operators. Therefore we choose the following gross variables

$$
P_{\mathbf{p}}=\left(\begin{array}{c}
\xi_{\mathbf{p} 1}^{\dagger} \xi_{\mathbf{p} 1} \\
\xi_{\mathbf{p},-1}^{\dagger} \xi_{\mathbf{p},-1} \\
\xi_{\mathbf{p} 1}^{\dagger} \xi_{\mathbf{p},-1} \\
\xi_{\mathbf{p},-1}^{\dagger} \xi_{\mathbf{p} 1}
\end{array}\right)
$$

the components of this vector being the second-quantization form of the above matrix

elements. The corresponding "quasi-equilibrium" or "coarse-grained" statistical operator (QSO) $)^{33,36}$ is given by

$$
\rho_{\mathbf{q}}=e^{-\Phi-\sum_{\mathbf{p}} F_{\mathbf{p}}^{\dagger} P_{\mathbf{p}}}=e^{-\Phi-\sum_{\mathbf{p}}\left(F_{\mathbf{p} 1} \xi_{\mathbf{p} 1}^{\dagger} \xi_{\mathbf{p} 1}+F_{\mathbf{p} 2} \xi_{\mathbf{p},-1}^{\dagger} \xi_{\mathbf{p},-1}+F_{\mathbf{p}} 3 \xi_{\mathbf{p} 1}^{\dagger} \xi_{\mathbf{p},-1}+F_{\mathbf{p} 3}^{*} \xi_{\mathbf{p},-1}^{\dagger} \xi_{\mathbf{p} 1}\right)},
$$

where

$$
\Phi=\ln \operatorname{Tr}\left[e^{-\sum_{\mathbf{p}}\left(F_{\mathbf{p} 1} \xi_{\mathbf{p} 1}^{\dagger} \xi_{\mathbf{p} 1}+F_{\mathbf{p} 2} \xi_{\mathbf{p},-1}^{\dagger} \xi_{\mathbf{p},-1}+F_{\mathbf{p} 3} \xi_{\mathbf{p} 1}^{\dagger} \xi_{\mathbf{p},-1}+F_{\mathbf{p} 3}^{*} \xi_{\mathbf{p},-1}^{\dagger} \xi_{\mathbf{p} 1}\right)}\right]
$$


is the generalized Masseu-Plank function. As at the equilibrium, $F_{\mathbf{p}}^{\dagger}$ are parameters conjugated to $P_{\mathbf{p}}$ in the sense that $33,34,35,36$

$$
\left\langle P_{\mathbf{p}}\right\rangle_{\mathrm{q}}=-\frac{\delta \Phi}{\delta F_{\mathbf{p}}^{\dagger}} .
$$

This QSO is second-quantization representation form of the general density matrix in the which allows for Wick rules. Following from it explicit connection between $\left\langle P_{\mathbf{p}}\right\rangle$ and $F_{\mathbf{p}}$, however, bears no new information.

To obtain GKE one uses the closure condition $\left\langle P_{\mathbf{p}}\right\rangle_{\mathbf{q}}=\left\langle P_{\mathbf{p}}\right\rangle$ assumed $\underline{33}, \underline{36}$ only for the gross variables, which results in

$$
\frac{\partial}{\partial t}\langle P\rangle_{\mathrm{q}}=i\left\langle\left[\mathcal{H}_{0}+\mathcal{H}_{\mathrm{int}}, P\right]\right\rangle
$$

where the averaging in the right-hand sides is performed over NSO obtained from QSO via an explicit formal prescription ${ }^{33,36}$. In our case this averaging is also to incorporate one over the impurity configurations. Note that in all known cases with weak interaction the operators $P_{\mathbf{p}}$ obey closed microscopic dynamics with the unperturbed Hamiltonian

$$
\left[\mathcal{H}_{0}, P_{\mathbf{p}}\right]=\sum_{\mathbf{q}} \omega_{\mathbf{p q}} P_{\mathbf{q}}
$$

where $\omega_{\mathbf{p q}}$ is a known matrix. It can be shown that in our case Eq. (27) sustains even if $\mathcal{H}_{0}$ includes the interaction with an electric field $\mathbf{E}$ along the $x$-axis

$$
\mathcal{H}_{\mathrm{ef}}=-e \mathbf{E} \cdot \mathbf{R}=-e E \sum_{\mathbf{p}, m= \pm 1}\left(i \xi_{\mathbf{p} m}^{\dagger} \nabla \xi_{\mathbf{p} m}-\frac{\sin \phi_{\mathbf{p}}}{2 p} \xi_{\mathbf{p} m}^{\dagger} \xi_{\mathbf{p},-m}\right)
$$

At that occurrence, the matrix $\omega_{\mathbf{p q}}$ contains linear in $E$ off-diagonal elements, some of which involve the gradients of the momentum-conservation delta function.

In the second-order approximation with respect to $\mathcal{H}_{\text {int }}$ Eq. (26) can be transformed $33,35,36$ to GKE, which have the following form common to all applications

$$
\frac{\partial\left\langle P_{\mathbf{p}}\right\rangle}{\partial t}=i \sum_{\mathbf{q}} \omega_{\mathbf{p q}}\left\langle P_{\mathbf{q}}\right\rangle+\mathcal{J}_{\mathbf{p}}^{(1)}+\mathcal{J}_{\mathbf{p}}^{(2)}
$$

where the generalized collision integrals of the first and second orders are given by $\underline{33,35,36}$

$$
\mathcal{J}_{\mathbf{p}}^{(1)}=i\left\langle\left[\mathcal{H}_{\text {int }}, P_{\mathbf{p}}\right]\right\rangle_{\mathbf{q}}
$$

and

$$
\mathcal{J}_{\mathbf{p}}^{(2)}=\lim _{\varepsilon \rightarrow+0} \int_{-\infty}^{0} e^{\varepsilon t} d t\left\langle\left[\mathcal{H}_{\text {int }}(t),\left[P_{\mathbf{p}}, \mathcal{H}_{\text {int }}\right]+i \sum_{\mathbf{l}} \frac{\delta \mathcal{J}_{\mathbf{p}}^{(1)}}{\delta\left\langle P_{\mathbf{l}}\right\rangle} P_{\mathbf{l}}\right]\right\rangle_{\mathbf{q}}
$$


respectively. Here the dependence of the interaction Hamiltonian on time $t$ is according to Heisenberg picture with $\mathcal{H}_{0}$ which, in addition to the kinetic energy, may include $\mathcal{H}_{\text {ef }}$. The term in Eq. (31), which involves $\mathcal{J}_{\mathbf{p}}^{(1)}$, leads to cancelling possible contributions that diverge in the thermodynamic limit $S \rightarrow \infty$ out of $\mathcal{J}_{\mathbf{p}}^{(2)}$. This is fair analog (in the Born approximation) to "connected-diagrams" statement in the diagram techniques. $\underline{30,31,32}$

\section{B. Average current density}

Using Eq.(15) the average current density can be expressed via the basic averages $\left\langle P_{\mathbf{p}}\right\rangle$ as follows

$$
j_{x}=\frac{\left\langle J_{x}\right\rangle}{S}=\frac{e v}{(2 \pi)^{2}} \int\left[\left(f_{\mathbf{p} 1}-f_{\mathbf{p},-1}\right) \cos \phi_{\mathbf{p}}+2 \operatorname{Im}\left(g_{\mathbf{p} 1}\right) \sin \phi_{\mathbf{p}}\right] d^{2} p
$$

where, by definition,

$$
f_{\mathbf{p} s}=\left\langle\xi_{\mathbf{p} s}^{\dagger} \xi_{\mathbf{p} s}\right\rangle, g_{\mathbf{p} s}=\left\langle\xi_{\mathbf{p} s}^{\dagger} \xi_{\mathbf{p},-s}\right\rangle=g_{\mathbf{p},-s}^{*}
$$

Let us now introduce the electron and hole distribution functions

$$
n_{\mathbf{p}}=f_{\mathbf{p} 1}, p_{\mathbf{p}}=1-f_{\mathbf{p},-1}
$$

which are, of course, real, and "anomalous" distribution function $g_{\mathbf{p}}=g_{\mathbf{p} 1}$, which is complex in general. In the terms of these functions Eq. (32) is written as follows

$$
\begin{aligned}
j_{x} & =\frac{e v}{(2 \pi)^{2}} \int\left[\left(n_{\mathbf{q}}+p_{\mathbf{q}}\right) \cos \phi_{\mathbf{q}}+2 \operatorname{Im}\left(g_{\mathbf{q}}\right) \sin \phi_{\mathbf{q}}\right] d^{2} q \\
& \equiv \frac{e v}{(2 \pi)^{2}} \int\left[N_{\mathbf{q}} \cos \phi_{\mathbf{q}}+2 \operatorname{Im}\left(g_{\mathbf{q}}\right) \sin \phi_{\mathbf{q}}\right] d^{2} q .
\end{aligned}
$$

\section{Derivation details}

Let us now specify Eqs. (29) - (31) for graphene. To this end, consider all the prerequisites of the calculations required. Using Eq. (21), we find

$$
\begin{aligned}
\mathcal{H}_{\mathrm{int}} & =S^{-1} \sum_{\mathbf{l}^{\prime}} V\left(\mathbf{l}-\mathbf{l}^{\prime}\right) \Xi_{\mathbf{l}}^{\dagger}\left(\begin{array}{cc}
\cos \frac{\phi_{\mathbf{1}}-\phi_{\mathbf{1}^{\prime}}}{2} & i \sin \frac{\phi_{\mathbf{1}}-\phi_{\mathbf{1}^{\prime}}}{2} \\
i \sin \frac{\phi_{\mathbf{1}}-\phi_{\mathbf{1}^{\prime}}}{2} & \cos \frac{\phi_{\mathbf{1}}-\phi_{\mathbf{1}^{\prime}}}{2}
\end{array}\right) \Xi_{\mathbf{l}^{\prime}} \\
& =S^{-1} \sum_{\mathbf{l} \mathbf{1}^{\prime}, m= \pm 1} V\left(\mathbf{l}-\mathbf{l}^{\prime}\right)\left(\cos \frac{\phi_{\mathbf{l}}-\phi_{\mathbf{l}^{\prime}}}{2} \xi_{\mathbf{l}, m}^{\dagger} \xi_{\mathbf{l}^{\prime}, m}++i \sin \frac{\phi_{\mathbf{l}}-\phi_{\mathbf{l}^{\prime}}}{2} \xi_{\mathbf{l}, m}^{\dagger} \xi_{\mathbf{l}^{\prime},-m}\right)
\end{aligned}
$$


and so

$$
\begin{aligned}
\mathfrak{L} & =\int_{-\infty}^{0} e^{\varepsilon t} \mathcal{H}_{\mathrm{int}}(t) d t \\
& =S^{-1} \sum_{\mathbf{l l}^{\prime}, m= \pm 1} V\left(\mathbf{l}-\mathbf{l}^{\prime}\right)\left[\frac{\cos \frac{\phi_{1}-\phi_{\mathbf{l}^{\prime}}}{2}}{\varepsilon+i m\left(\epsilon_{l}-\epsilon_{l^{\prime}}\right)} \xi_{\mathbf{l}_{m}}^{\dagger} \xi_{\mathbf{l}^{\prime} m}+\frac{i \sin \frac{\phi_{1}-\phi_{\mathbf{l}^{\prime}}}{2}}{\varepsilon+i m\left(\epsilon_{l}+\epsilon_{l^{\prime}}\right)} \xi_{\mathbf{l}_{m}}^{\dagger} \xi_{\mathbf{l}^{\prime},-m}\right] .
\end{aligned}
$$

Consider further the commutators of the gross-variable operators with $\mathcal{H}_{0}$. By a straightforward calculation we obtain

$$
\begin{aligned}
{\left[\mathcal{H}_{0}, \xi_{\mathbf{p} s}^{\dagger} \xi_{\mathbf{p} s}\right] } & =\left[\sum_{\mathbf{q}, n= \pm 1}\left(n v p \xi_{\mathbf{q} n}^{\dagger} \xi_{\mathbf{q} n}-i e E \xi_{\mathbf{q} n}^{\dagger} \frac{\partial \xi_{\mathbf{q} n}}{\partial q_{x}}+\frac{e E}{2 q} \sin \phi_{\mathbf{q}} \xi_{\mathbf{q} n}^{\dagger} \xi_{\mathbf{q},-n}\right), \xi_{\mathbf{p} s}^{\dagger} \xi_{\mathbf{p} s}\right] \\
& =i e E \frac{\partial}{\partial p_{x}}\left(\xi_{\mathbf{p} s}^{\dagger} \xi_{\mathbf{p} s}\right)-\frac{e E}{2 p}\left(\xi_{\mathbf{p} s}^{\dagger} \xi_{\mathbf{p},-s}-\xi_{\mathbf{p},-s}^{\dagger} \xi_{\mathbf{p} s}\right) \sin \phi_{\mathbf{p}}
\end{aligned}
$$

and

$$
\begin{aligned}
{\left[\mathcal{H}_{0}, \xi_{\mathbf{p} s}^{\dagger} \xi_{\mathbf{p},-s}\right] } & =\left[\sum_{\mathbf{q}, n= \pm 1}\left(n v p \xi_{\mathbf{q} n}^{\dagger} \xi_{\mathbf{q} n}-i e E \xi_{\mathbf{q} n}^{\dagger} \frac{\partial \xi_{\mathbf{q} n}}{\partial q_{x}}+\frac{e E}{2 q} \sin \phi_{\mathbf{q}} \xi_{\mathbf{q} n}^{\dagger} \xi_{\mathbf{q},-n}\right), \xi_{\mathbf{p} s}^{\dagger} \xi_{\mathbf{p},-s}\right] \\
& =2 \operatorname{svp} \xi_{\mathbf{p} s}^{\dagger} \xi_{\mathbf{p},-s}+i e E \frac{\partial}{\partial p_{x}}\left(\xi_{\mathbf{p} s}^{\dagger} \xi_{\mathbf{p},-s}\right)-\frac{e E}{2 p}\left(\xi_{\mathbf{p} s}^{\dagger} \xi_{\mathbf{p}, s}-\xi_{\mathbf{p},-s}^{\dagger} \xi_{\mathbf{p},-s}\right) \sin \phi_{\mathbf{p}}
\end{aligned}
$$

Hence the "precession" terms in the right-hand side of GKE, see Eq. (29), are

$$
\begin{aligned}
i \sum_{\mathbf{q}} \omega_{\mathbf{p} s, \mathbf{q} s}\left\langle\xi_{\mathbf{p} s}^{\dagger} \xi_{\mathbf{q} s}\right\rangle_{q} & =-e E \frac{\partial f_{\mathbf{p} s}}{\partial p_{x}}+\frac{e E \sin \phi_{\mathbf{p}}}{p} \operatorname{Im} g_{\mathbf{p} s}, \\
i \sum_{\mathbf{q}} \omega_{\mathbf{p} s, \mathbf{q}-s}\left\langle\xi_{\mathbf{p} s}^{\dagger} \xi_{\mathbf{q} s}\right\rangle_{q} & =2 i \operatorname{svp} g_{\mathbf{p} s}-e E \frac{\partial g_{\mathbf{p} s}}{\partial p_{x}}-i \frac{e E \sin \phi_{\mathbf{p}}}{2 p}\left(f_{\mathbf{p} s}-f_{\mathbf{p},-s}\right),
\end{aligned}
$$

where we have used the notations introduced in Eq. (33).

To calculate collision integrals for the "normal" distribution functions $f_{\mathbf{p} s}$ and the anomalous ones $g_{\mathbf{p} s}$ we are to perform the commutation twice - first time to commute the grossvariables operators with $\mathcal{H}_{\text {int }}$ to obtain $\mathcal{J}_{\mathbf{p}}^{(1)}$ and the second to commute the result of the first commutation with $\mathfrak{L}$ to obtain $\mathcal{J}_{\mathbf{p}}^{(2)}$. Following this route we get

$$
\begin{aligned}
{\left[\xi_{\mathbf{p} s}^{\dagger} \xi_{\mathbf{p} s}, \mathcal{H}_{\text {int }}\right] } & =S^{-1} \sum_{\mathbf{q q}^{\prime}} V\left(\mathbf{q}-\mathbf{q}^{\prime}\right)\left[\cos \frac{\phi_{\mathbf{q}}-\phi_{\mathbf{q}^{\prime}}}{2}\left(\delta_{\mathbf{p}, \mathbf{q}}-\delta_{\mathbf{p}, \mathbf{q}^{\prime}}\right) \xi_{\mathbf{q}_{s} s}^{\dagger} \xi_{\mathbf{q}^{\prime} s}\right. \\
& \left.+i \sin \frac{\phi_{\mathbf{q}}-\phi_{\mathbf{q}^{\prime}}}{2}\left(\delta_{\mathbf{p}, \mathbf{q}} \xi_{\mathbf{q}_{s}}^{\dagger} \xi_{\mathbf{q}^{\prime},-s}-\delta_{\mathbf{p}, \mathbf{q}^{\prime}} \xi_{\mathbf{q},-s}^{\dagger} \xi_{\mathbf{q}^{\prime}, s}\right)\right] .
\end{aligned}
$$

Averaging this expression over QSO gives $\mathcal{J}_{\mathbf{p}}^{(1)}\left[f_{s}\right]=0$. Then, performing the second commutation using Eqs. (37) and (39), after straightforward calculations we obtain the second- 
order collision integral for $f_{\mathbf{p} s}$

$$
\begin{aligned}
\mathcal{J}_{\mathbf{p}}^{(2)}\left[f_{s}\right] & =S^{-2} \sum_{\mathbf{q}}|V(\mathbf{p}-\mathbf{q})|^{2}\left\{s \sin \left(\phi_{\mathbf{p}}-\phi_{\mathbf{q}}\right) \operatorname{Re}\left(g_{\mathbf{q}}\right)\left(\frac{1}{\epsilon_{p}+\epsilon_{q}}+\frac{1}{\epsilon_{q}-\epsilon_{p}}\right)\right. \\
& \left.-\pi\left[2 \cos ^{2} \frac{\phi_{\mathbf{p}}-\phi_{\mathbf{q}}}{2}\left(f_{\mathbf{p} s}-f_{\mathbf{q} s}\right)+s \sin \left(\phi_{\mathbf{p}}-\phi_{\mathbf{q}}\right) \operatorname{Im}\left(g_{\mathbf{q}}\right)\right] \delta\left(\epsilon_{p}-\epsilon_{q}\right)\right\} .
\end{aligned}
$$

Further we have

$$
\begin{aligned}
{\left[\xi_{\mathbf{p} s}^{\dagger} \xi_{\mathbf{p},-s}, \mathcal{H}_{\mathrm{int}}\right] } & =S^{-1} \sum_{\mathbf{q q}^{\prime}} V\left(\mathbf{q}-\mathbf{q}^{\prime}\right)\left[\cos \frac{\phi_{\mathbf{q}}-\phi_{\mathbf{q}^{\prime}}}{2}\left(\delta_{\mathbf{p q}}-\delta_{\mathbf{p q}^{\prime}}\right) \xi_{\mathbf{q} s}^{\dagger} \xi_{\mathbf{q}^{\prime},-s}\right. \\
& \left.+i \sin \frac{\phi_{\mathbf{q}}-\phi_{\mathbf{q} \mathbf{q}^{\prime}}}{2}\left(\delta_{\mathbf{p q}} \xi_{\mathbf{q}^{s}}^{\dagger} \xi_{\mathbf{q}^{\prime} s}-\delta_{\mathbf{p q ^ { \prime }}} \xi_{\mathbf{q},-s}^{\dagger} \xi_{\mathbf{q}^{\prime},-s}\right)\right]
\end{aligned}
$$

Making the second commutation with the use of Eqs. (37) and (41), after straightforward calculations we obtain the second-order collision integral for $g_{\mathbf{p} s}$

$$
\begin{aligned}
\mathcal{J}_{\mathbf{p}}^{(2)}\left[g_{s}\right]= & -S^{-2} \sum_{\mathbf{q q}^{\prime}}|V(\mathbf{p}-\mathbf{q})|^{2}\left\{2 \cos ^{2} \frac{\phi_{\mathbf{p}}-\phi_{\mathbf{q}}}{2} \times\right. \\
& {\left[\left(g_{\mathbf{p} s}-g_{\mathbf{q} s}\right) \pi \delta\left(\epsilon_{p}-\epsilon_{q}\right)+i s \frac{g_{\mathbf{p} s}+g_{\mathbf{q} s}}{\epsilon_{q}-\epsilon_{p}}\right]-\frac{i}{2} \sin \left(\phi_{\mathbf{p}}-\phi_{\mathbf{q}}\right) \times } \\
& \left(f_{\mathbf{q} s}+f_{\mathbf{q},-s}\right)\left[\pi \delta\left(\epsilon_{p}-\epsilon_{q}\right)-i s \frac{1}{\epsilon_{q}-\epsilon_{p}}\right]+ \\
& \left.\frac{s}{2} \sin \left(\phi_{\mathbf{p}}-\phi_{\mathbf{q}}\right) \frac{f_{\mathbf{q} s}-f_{\mathbf{q},-s}}{\epsilon_{p}+\epsilon_{q}}-2 i s \sin ^{2} \frac{\phi_{\mathbf{p}}-\phi_{\mathbf{q}}}{2} \frac{g_{\mathbf{p} s}+g_{\mathbf{q} s}^{*}}{\epsilon_{p}+\epsilon_{q}}\right\} .
\end{aligned}
$$

This equation describes the Zitterbewegung effects, that is, creation of electron-hole pairs during the charge carrier propagation. Note that in both Eq. (40) and (42) the configurational average of the potential Fourier transforms squared is implied.

Putting together Eqs.(38), (40) and (42) we can write the final set of GKE for the "normal" and "anomalous" distribution functions. It is more convenient, however, to transform these GKE to a system of equations for the functions

$$
D_{\mathbf{p}}=\sum_{s= \pm 1} f_{\mathbf{p} s}-1=n_{\mathbf{p}}-p_{\mathbf{p}}, N_{\mathbf{p}}=\sum_{s= \pm 1} s f_{\mathbf{p} s}+1=n_{\mathbf{p}}+p_{\mathbf{p}}
$$

and for $g_{\mathbf{p}}$. Let us remind that the latter and $N_{\mathbf{p}}$ define the average current, see Eq. (32). We have for $D_{\mathbf{p}}$

$$
\frac{\partial D_{\mathbf{p}}}{\partial t}+e E \frac{\partial D_{\mathbf{p}}}{\partial p_{x}}=-\frac{2 \pi}{S^{2}} \sum_{\mathbf{q}}|V(\mathbf{p}-\mathbf{q})|^{2} \cos ^{2} \frac{\phi_{\mathbf{p}}-\phi_{\mathbf{q}}}{2}\left(D_{\mathbf{p}}-D_{\mathbf{q}}\right) \delta\left(\epsilon_{p}-\epsilon_{q}\right)
$$


which does not involve $g_{\mathbf{p}}$ at all, while the equation for $N_{\mathbf{p}}$ reads

$$
\begin{aligned}
& \frac{\partial N_{\mathbf{p}}}{\partial t}+e E \frac{\partial N_{\mathbf{p}}}{\partial p_{x}}-\frac{2 e E \sin \phi_{\mathbf{p}}}{p} \operatorname{Im} g_{\mathbf{p}} \\
& =\frac{2 \pi}{S^{2}} \sum_{\mathbf{q}}|V(\mathbf{p}-\mathbf{q})|^{2}\left\{\pi^{-1} \sin \left(\phi_{\mathbf{p}}-\phi_{\mathbf{q}}\right) \operatorname{Re}\left(g_{\mathbf{q}}\right)\left(\frac{1}{\epsilon_{q}+\epsilon_{p}}+\frac{1}{\epsilon_{q}-\epsilon_{p}}\right)\right. \\
& \left.-\left[\cos ^{2} \frac{\phi_{\mathbf{p}}-\phi_{\mathbf{q}}}{2}\left(N_{\mathbf{p}}-N_{\mathbf{q}}\right)+\sin \left(\phi_{\mathbf{p}}-\phi_{\mathbf{q}}\right) \operatorname{Im}\left(g_{\mathbf{q}}\right)\right] \delta\left(\epsilon_{p}-\epsilon_{q}\right)\right\}
\end{aligned}
$$

Finally, the equation for the complex function $g_{\mathbf{p}}$ proves the following

$$
\begin{aligned}
& \frac{\partial g_{\mathbf{p}}}{\partial t}-2 i v p g_{\mathbf{p}}+e E \frac{\partial g_{\mathbf{p}}}{\partial p_{x}}+i \frac{e E}{2 p}\left(N_{\mathbf{p}}-1\right) \sin \phi_{\mathbf{p}}= \\
& -\frac{\pi}{S^{2}} \sum_{\mathbf{q}}|V(\mathbf{p}-\mathbf{q})|^{2}\left\{-\frac{i}{2} \sin \left(\phi_{\mathbf{p}}-\phi_{\mathbf{q}}\right) D_{\mathbf{q}}\left[\delta\left(\epsilon_{p}-\epsilon_{q}\right)+\frac{i}{\pi} \frac{1}{\epsilon_{p}-\epsilon_{q}}\right]\right. \\
& +2 \cos ^{2} \frac{\phi_{\mathbf{p}}-\phi_{\mathbf{q}}}{2}\left[\left(g_{\mathbf{p}}-g_{\mathbf{q}}\right) \delta\left(\epsilon_{p}-\epsilon_{q}\right)+\frac{i}{\pi} \frac{g_{\mathbf{p}}+g_{\mathbf{q}}}{\epsilon_{q}-\epsilon_{p}}\right] \\
& \left.+\frac{1}{2 \pi} \frac{N_{\mathbf{q}}}{\epsilon_{p}+\epsilon_{q}} \sin \left(\phi_{\mathbf{p}}-\phi_{\mathbf{q}}\right)-\frac{2 i}{\pi} \frac{g_{\mathbf{p}}+g_{\mathbf{q}}^{*}}{\epsilon_{p}+\epsilon_{q}} \sin ^{2} \frac{\phi_{\mathbf{p}}-\phi_{\mathbf{q}}}{2}\right\} .
\end{aligned}
$$

\section{THE LINEAR RESPONSE REGIME IN ELECTRIC FIELD}

In general, Eqs. (44) - (46) are quite complicated. Further we will consider only the regime of linear response, that is, the case of weak electric field. We will restrict ourselves also by the case of stationary field and neglect its effect on the collision integral. It can be shown that linear in $E$ corrections to $\mathcal{J}_{\mathbf{p}}^{(2)}$ restore some second order terms of the perturbation expansion of the exact field term considered in the Luttinger-Kohn formalism ${ }^{29}$, which lies out of our scope.

At $E=0$, like the classical kinetic equation, Eqs. (44) - (46) have equilibrium solution. In our case it is three arbitrary function of the energy $\epsilon_{p}=v p$. To develop meaningful linearization of GKE and the current for small $E$, we assume, following Ref. 29, presence of a thermostat which role is only to establish true equilibrium with a temperature $T$. Under

this condition the equilibrium distribution functions $n_{0 \mathbf{p}}$ and $p_{0 \mathbf{p}}$ become, of course, the electrons and holes Fermi functions, respectively, with unique chemical potential $\mu$. Now let us consider the linearization of GKE derived above in detail. 


\section{A. Linearized equation for $D_{\mathbf{p}}$}

Replacing in the field term of Eq.(44) the distribution functions by their equilibrium value and linearizing the corresponding collision integral, we obtain the following equation

$$
e E \frac{\partial D_{0}\left(\epsilon_{p}\right)}{\partial p_{x}}=-\frac{2 \pi}{S^{2}} \sum_{\mathbf{q}}|V(\mathbf{p}-\mathbf{q})|^{2} \cos ^{2} \frac{\phi_{\mathbf{p}}-\phi_{\mathbf{q}}}{2}\left(\delta D_{\mathbf{p}}-\delta D_{\mathbf{q}}\right) \delta\left(\epsilon_{p}-\epsilon_{q}\right),
$$

where

$$
D_{0}(\epsilon)=\frac{1}{e^{\frac{\epsilon-\mu}{T}}+1}-\frac{1}{e^{\frac{\epsilon+\mu}{T}}+1},
$$

Eq. (47) is quite similar to the classical kinetic equation and so is routinely solved exactly. The solution reads

$$
\delta D_{\mathbf{p}}=-e \operatorname{Ev\tau }\left(\epsilon_{p}\right) \frac{\partial D_{0}\left(\epsilon_{p}\right)}{\partial \epsilon_{p}} \cos \phi_{\mathbf{p}}
$$

$\tau\left(\epsilon_{p}\right)$ being standard elastic transport relaxation time given by

$$
\begin{aligned}
\frac{1}{\tau\left(\epsilon_{p}\right)} & =\frac{\pi}{S^{2}} \sum_{\mathbf{q}}|V(\mathbf{p}-\mathbf{q})|^{2} \sin ^{2}\left(\phi_{\mathbf{p}}-\phi_{\mathbf{q}}\right) \delta\left(\epsilon_{p}-\epsilon_{q}\right)= \\
& =\frac{\pi \epsilon_{p}}{(2 \pi v)^{2}} N_{\mathrm{imp}} \int_{0}^{2 \pi}\left|U\left(2 p \sin \frac{\phi}{2}\right)\right|^{2} \sin ^{2} \phi d \phi,
\end{aligned}
$$

where $N_{\text {imp }}=c / \Omega$ is the impurity concentration per the area unit, $c$ and $\Omega$ being atomic fraction of impurities and the graphene crystal cell area, respectively, and $U(|\mathbf{q}|)$ is the Fourier transform of one-impurity potential. In deriving Eq. (150) and what follows we

adopted that $c \ll 1$. The factor $\sin ^{2} \phi$ instead of $\operatorname{standard} 20$ one $1-\cos \phi$ is a consequence of the chiral character of charge carriers which leads to the suppressions of back scattering 10,16 .

\section{B. Linearized equations for $N_{\mathbf{p}}$ and $g_{\mathbf{p}}$}

Replacing in the field terms of Eqs. (45) and (46) the distribution functions by their equilibrium value and linearizing the corresponding collision integrals, we arrive at the following system of coupled equations

$$
\begin{aligned}
& e E \frac{\partial N_{0}\left(\epsilon_{p}\right)}{\partial p_{x}}-\frac{2 e E \sin \phi_{\mathbf{q}}}{q} \operatorname{Im} g_{0}\left(\epsilon_{p}\right)= \\
& \frac{2 \pi}{S^{2}} \sum_{\mathbf{q}}|V(\mathbf{p}-\mathbf{q})|^{2}\left\{\sin \left(\phi_{\mathbf{p}}-\phi_{\mathbf{q}}\right)\left(\frac{1}{\epsilon_{q}+\epsilon_{p}}+\frac{1}{\pi} \frac{1}{\epsilon_{q}-\epsilon_{p}}\right) \operatorname{Re} \delta g_{\mathbf{q}}\right. \\
& \left.-\left[\cos ^{2} \frac{\phi_{\mathbf{p}}-\phi_{\mathbf{q}}}{2}\left(\delta N_{\mathbf{p}}-\delta N_{\mathbf{q}}\right)+\sin \left(\phi_{\mathbf{p}}-\phi_{\mathbf{q}}\right) \operatorname{Im} \delta g_{\mathbf{q}}\right] \delta\left(\epsilon_{p}-\epsilon_{q}\right)\right\}
\end{aligned}
$$


and

$$
\begin{aligned}
& -2 i \epsilon_{p} \delta g_{\mathbf{p}}+e E \frac{\partial g_{0}\left(\epsilon_{p}\right)}{\partial p_{x}}+i \frac{e E \sin \phi_{\mathbf{p}}}{2 p}\left[N_{0}\left(\epsilon_{p}\right)-1\right]= \\
& -\frac{\pi}{S^{2}} \sum_{\mathbf{q}}|V(\mathbf{p}-\mathbf{q})|^{2}\left\{-\frac{i}{2} \delta D_{\mathbf{q}}\left[\delta\left(\epsilon_{p}-\epsilon_{q}\right)+\frac{i}{\pi} \frac{1}{\epsilon_{p}-\epsilon_{q}}\right] \sin \left(\phi_{\mathbf{p}}-\phi_{\mathbf{q}}\right)\right. \\
& +2\left[\left(\delta g_{\mathbf{p}}-\delta g_{\mathbf{q}}\right) \delta\left(\epsilon_{p}-\epsilon_{q}\right)+\frac{i}{\pi} \frac{\delta g_{\mathbf{p}}+\delta g_{\mathbf{q}}}{\epsilon_{q}-\epsilon_{p}}\right] \cos ^{2} \frac{\phi_{\mathbf{p}}-\phi_{\mathbf{q}}}{2} \\
& \left.+\frac{1}{2 \pi} \frac{\delta N_{\mathbf{q}}}{\epsilon_{p}+\epsilon_{q}} \sin \left(\phi_{\mathbf{p}}-\phi_{\mathbf{q}}\right)-\frac{2 i}{\pi} \frac{\delta g_{\mathbf{p}}+\delta g_{\mathbf{q}}^{*}}{\epsilon_{p}+\epsilon_{q}} \sin ^{2} \frac{\phi_{\mathbf{p}}-\phi_{\mathbf{q}}}{2}\right\},
\end{aligned}
$$

where

$$
N_{0}(\epsilon)=\frac{1}{e^{\frac{\epsilon-\mu}{T}}+1}+\frac{1}{e^{\frac{\epsilon+\mu}{T}}+1},
$$

and $g_{0}\left(\epsilon_{p}\right)$ is an equilibrium "anomalous" distribution function satisfying the equation

$$
\begin{aligned}
& 2 i \epsilon_{p} g_{0}\left(\epsilon_{p}\right)=\frac{\pi}{S^{2}} \sum_{\mathbf{q}}|V(\mathbf{p}-\mathbf{q})|^{2}\left\{-\frac{i}{2} D_{0}\left(\epsilon_{q}\right)\left[\delta\left(\epsilon_{p}-\epsilon_{q}\right)+\frac{i}{\pi} \frac{1}{\epsilon_{p}-\epsilon_{q}}\right] \sin \left(\phi_{\mathbf{p}}-\phi_{\mathbf{q}}\right)+\right. \\
& \left.\frac{2 i}{\pi}\left[\frac{g_{0}\left(\epsilon_{p}\right)+g_{0}\left(\epsilon_{q}\right)}{\epsilon_{q}-\epsilon_{p}} \cos ^{2} \frac{\phi_{\mathbf{p}}-\phi_{\mathbf{q}}}{2}-\frac{g_{0}\left(\epsilon_{p}\right)+g_{0}^{*}\left(\epsilon_{q}\right)}{\epsilon_{p}+\epsilon_{q}} \sin ^{2} \frac{\phi_{\mathbf{p}}-\phi_{\mathbf{q}}}{2}\right]+\frac{1}{2 \pi} \frac{N_{0}\left(\epsilon_{q}\right)}{\epsilon_{p}+\epsilon_{q}} \sin \left(\phi_{\mathbf{p}}-\phi_{\mathbf{q}}\right)\right\} .
\end{aligned}
$$

Because

$$
\int_{0}^{2 \pi}|V(\mathbf{p}-\mathbf{q})|^{2} \sin \left(\phi_{\mathbf{p}}-\phi_{\mathbf{q}}\right) d \phi_{\mathbf{q}} \propto \int_{0}^{2 \pi}\left|U\left(\sqrt{p^{2}-2 p q \cos \chi+q^{2}}\right)\right|^{2} \sin \chi d \chi \equiv 0
$$

the terms containing $D_{0}\left(\epsilon_{q}\right)$ and $N_{0}\left(\epsilon_{q}\right)$ in the above equation for $g_{0}\left(\epsilon_{p}\right)$ give zero contributions. As a result, if even non-zero $g_{0}(\epsilon)$ is purely real. In contrast with $N_{0}(\epsilon)$ and $D_{0}(\epsilon)$, this real function has no influence on the non-equilibrium $\delta N_{\mathbf{p}}$ and $\delta g_{\mathbf{p}}$ and so drops out of our treatment.

Let us search the functions $\delta N_{\mathbf{p}}$ and $\delta g_{\mathbf{p}}$ in the form

$$
\delta N_{\mathbf{p}}=-e E \nu\left(\epsilon_{p}\right) \cos \phi_{\mathbf{p}}
$$

and

$$
\delta g_{\mathbf{p}}=e E \gamma\left(\epsilon_{p}\right) \sin \phi_{\mathbf{p}}
$$

respectively. Substituting these forms into expression for the average current (35) yields the following expression for the conductivity

$$
\sigma=\frac{e^{2}}{2 \pi v} \int_{0}^{\infty}\left[\operatorname{Im} \gamma(\epsilon)-\frac{\nu(\epsilon)}{2}\right] \epsilon d \epsilon .
$$


Substitute further Eqs.(54) and (55) into Eq.(51). Taking into account that $\operatorname{Im} g_{0}\left(\epsilon_{p}\right)=0$, we manage to show that all non-zeroing terms of the resulting equation contain common factor $\cos \phi_{\mathbf{p}}$. Dividing by this factor we get the first linear-response GKE

$$
v \frac{\partial N_{0}\left(\epsilon_{p}\right)}{\partial \epsilon_{p}}=\frac{\nu\left(\epsilon_{p}\right)+2 \operatorname{Im} \gamma\left(\epsilon_{p}\right)}{\tau\left(\epsilon_{p}\right)}-2 \int_{0}^{\infty} \Gamma_{0}(p, q)\left(\frac{1}{q+p}+\frac{1}{q-p}\right) \operatorname{Re} \gamma\left(\epsilon_{q}\right) d q,
$$

where the kernel in the integral term is given by

$$
\Gamma_{0}(p, q)=\frac{\epsilon_{q}}{(2 \pi v)^{2}} N_{\mathrm{imp}} \int_{0}^{2 \pi}\left|U\left(\sqrt{p^{2}-2 p q \cos \phi+q^{2}}\right)\right|^{2} \sin ^{2} \phi d \phi
$$

It is expedient to note that

$$
\Gamma_{0}(p, p)=\frac{1}{\pi \tau\left(\epsilon_{p}\right)}
$$

Performing quite similar transformations in the "anomalous" kinetic equation, Eq.(52), we find that all non-zeroing terms of the resulting equation are proportional to $\sin \phi_{\mathbf{p}}$. Cancelling this common factor, we obtain the second linear-response GKE

$$
\begin{aligned}
& -2 i \epsilon_{p} \gamma\left(\epsilon_{p}\right)=\frac{i v}{2}\left[\frac{1-N_{0}\left(\epsilon_{p}\right)}{\epsilon_{p}}-\frac{\partial D_{0}\left(\epsilon_{p}\right)}{\partial \epsilon_{p}}\right] \\
& -\frac{N_{\text {imp }}}{(2 \pi)^{2}} \int|U(\mathbf{p}-\mathbf{q})|^{2}\left\{-\frac{1}{2} \sin ^{2}\left(\phi_{\mathbf{p}}-\phi_{\mathbf{q}}\right)\left[\frac{\partial D_{0}\left(\epsilon_{q}\right)}{\partial \epsilon_{q}} \frac{v \tau\left(\epsilon_{q}\right)}{\epsilon_{p}-\epsilon_{q}}+\frac{\nu\left(\epsilon_{p}\right)}{\epsilon_{p}+\epsilon_{q}}\right]\right. \\
& +\left[1+\cos \left(\phi_{\mathbf{q}}-\phi_{\mathbf{p}}\right)\right]\left[\gamma\left(\epsilon_{p}\right)\left(1-\cos \left(\phi_{\mathbf{q}}-\phi_{\mathbf{p}}\right)\right) \pi \delta\left(\epsilon_{p}-\epsilon_{q}\right)\right. \\
& \left.+i \frac{\gamma\left(\epsilon_{p}\right)+\gamma\left(\epsilon_{q}\right) \cos \left(\phi_{\mathbf{q}}-\phi_{\mathbf{p}}\right)}{\epsilon_{q}-\epsilon_{p}}\right] \\
& \left.-i\left[1-\cos \left(\phi_{\mathbf{q}}-\phi_{\mathbf{p}}\right)\right] \frac{\gamma\left(\epsilon_{p}\right)+\gamma^{*}\left(\epsilon_{q}\right) \cos \left(\phi_{\mathbf{q}}-\phi_{\mathbf{p}}\right)}{\epsilon_{p}+\epsilon_{q}}\right\} d^{2} q .
\end{aligned}
$$

\section{Linear-response GKE in the energy variable}

In what follows we will make overall use of the energy variable $\epsilon=v p$. Let us summarize linear-response GK, i.e Eqs. (57) and (60), in terms of $\epsilon$. We have

$$
v \frac{\partial N_{0}(\epsilon)}{\partial \epsilon}=\frac{\nu(\epsilon)}{\tau(\epsilon)}+\frac{2 \operatorname{Im} \gamma(\epsilon)}{\tau(\epsilon)}-2 \int_{0}^{\infty} \widetilde{\Gamma}_{0}(\epsilon, \omega)\left(\frac{1}{\omega+\epsilon}+\frac{1}{\omega-\epsilon}\right) \operatorname{Re} \gamma(\omega) d \omega
$$

and

$$
\begin{aligned}
& -2 i \epsilon \gamma(\epsilon)=\frac{i v}{2}\left[\frac{1-N_{0}(\epsilon)}{\epsilon}-\frac{\partial D_{0}(\epsilon)}{\partial \epsilon}\right]+\frac{1}{2} \int_{0}^{\infty} \widetilde{\Gamma}_{0}(\epsilon, \omega)\left[\frac{\nu(\omega)}{\epsilon+\omega}+\frac{\partial D_{0}(\omega)}{\partial \omega} \frac{v \tau(\omega)}{\epsilon-\omega}\right] d \omega \\
& -\frac{\gamma(\epsilon)}{\tau(\epsilon)}+i \Delta(\epsilon) \gamma(\epsilon)+i \int_{0}^{\infty}\left[\frac{\widetilde{\Gamma}_{+}(\epsilon, \omega)}{\epsilon-\omega} \gamma(\omega)+\frac{\widetilde{\Gamma}_{-}(\epsilon, \omega)}{\epsilon+\omega} \gamma^{*}(\omega)\right] d \omega,
\end{aligned}
$$


where the kernels are given by

$$
\begin{aligned}
& \widetilde{\Gamma}_{0}(\epsilon, \omega)=\frac{\omega}{(2 \pi v)^{2}} N_{\mathrm{imp}} \int_{0}^{2 \pi}\left|U\left(\frac{1}{v} \sqrt{\epsilon^{2}-2 \epsilon \omega \cos \phi+\omega^{2}}\right)\right|^{2} \sin ^{2} \phi d \phi \equiv \Gamma_{0}\left(\frac{\epsilon}{v}, \frac{\omega}{v}\right), \\
& \widetilde{\Gamma}_{ \pm}(\epsilon, \omega)=\frac{\omega}{(2 \pi v)^{2}} N_{\mathrm{imp}} \int_{0}^{2 \pi}\left|U\left(\frac{1}{v} \sqrt{\epsilon^{2}-2 \epsilon \omega \cos \phi+\omega^{2}}\right)\right|^{2}(1 \pm \cos \phi) \cos \phi d \phi
\end{aligned}
$$

and, using these kernels, two-particle energy shift by

$$
\Delta(\epsilon)=\int_{0}^{\infty}\left[\frac{\widetilde{\Gamma}_{0}(\epsilon, \omega)+\widetilde{\Gamma}_{+}(\epsilon, \omega)}{\epsilon-\omega}+\frac{\widetilde{\Gamma}_{0}(\epsilon, \omega)-\widetilde{\Gamma}_{-}(\epsilon, \omega)}{\omega+\epsilon}\right] d \omega .
$$

Let us introduce dimensionless kernels $\Phi_{\alpha}(\epsilon, \omega), \alpha=0, \pm$ by the following identity relations

$$
\widetilde{\Gamma}_{0}(\epsilon, \omega) \equiv \omega \Phi_{0}(\epsilon, \omega), \quad \widetilde{\Gamma}_{ \pm}(\epsilon, \omega) \equiv \pm \omega \Phi_{ \pm}(\epsilon, \omega),
$$

with which and Eq. (63) the formulas of these new kernels being straightforward. Note that, by construction of the kinetic equations, $\left|\Phi_{\alpha}(\epsilon, \omega)\right| \ll 1$. Making use of the kernels $\Phi_{\alpha}(\epsilon, \omega)$ and of new functions of the energy defined by

$$
\epsilon \nu(\epsilon)=v\left[2 f_{0}(\epsilon)+\frac{1}{\pi \Phi_{0}(\epsilon, \epsilon)} \frac{\partial N_{0}(\epsilon)}{\partial \epsilon}\right], \epsilon \gamma(\epsilon)=v f(\epsilon), f=f_{1}+i f_{2}
$$

we arrive at a modified conductivity expression and two coupled singular integral equations for $f_{0}(\epsilon)$ and $f(\epsilon)$. We have

$$
\sigma=\sigma_{\mathrm{B}}+\frac{e^{2}}{2 \pi} \int_{0}^{\infty}\left[f_{2}(\epsilon)-f_{0}(\epsilon)\right] d \epsilon
$$

where

$$
\sigma_{\mathrm{B}}=-\frac{e^{2}}{(2 \pi)^{2}} \int_{0}^{\infty} \frac{1}{\Phi_{0}(\epsilon, \epsilon)} \frac{\partial N_{0}(\epsilon)}{\partial \epsilon} d \epsilon
$$

is the classical Boltzmann conductivity ${ }^{20}$, with the Born impurity scattering cross-section modified due to chirality as noted above, in which of electrons and holes contribute additively, while the additional non-classical term is due to the Zitterbewegung. The integral equations are as follows. The first one is real and homogenous

$$
\pi \Phi_{0}(\epsilon, \epsilon)\left[f_{2}(\epsilon)+f_{0}(\epsilon)\right]-\int_{0}^{\infty} \Phi_{0}(\epsilon, \omega)\left(\frac{1}{\omega+\epsilon}+\frac{1}{\omega-\epsilon}\right) f_{1}(\omega) d \omega=0
$$

and the second equation is complex and inhomogeneous

$$
\begin{aligned}
& {\left[\Lambda(\epsilon)+i \pi \Phi_{0}(\epsilon, \epsilon)\right] f(\epsilon)-\int_{0}^{\infty}\left[\frac{\Phi_{+}(\epsilon, \omega)}{\omega-\epsilon} f(\omega)+\frac{\Phi_{-}(\epsilon, \omega)}{\omega+\epsilon} f^{*}(\omega)\right] d \omega} \\
& -i \int_{0}^{\infty} \frac{\Phi_{0}(\epsilon, \omega)}{\epsilon+\omega} f_{0}(\omega) d \omega=F(\epsilon),
\end{aligned}
$$


in which

$$
\Lambda(\epsilon)=2+\epsilon^{-1} \Delta(\epsilon)
$$

and $F(\epsilon)=F_{1}(\epsilon)+i F_{2}(\epsilon)$, where

$$
\begin{gathered}
F_{1}(\epsilon)=-\frac{1-N_{0}(\epsilon)}{2 \epsilon}+\frac{1}{2} \frac{\partial D_{0}(\epsilon)}{\partial \epsilon} \\
F_{2}(\epsilon)=\frac{1}{2 \pi} \int_{0}^{\infty} \frac{\Phi_{0}(\epsilon, \omega)}{\Phi_{0}(\omega, \omega)}\left[\frac{1}{\omega+\epsilon} \frac{\partial N_{0}(\omega)}{\partial \omega}-\frac{1}{\omega-\epsilon} \frac{\partial D_{0}(\omega)}{\partial \omega}\right] d \omega .
\end{gathered}
$$

Eq.(70) is, in turn, equivalent to two real equations for $f_{1}$ and $f_{2}$

$$
\Lambda(\epsilon) f_{1}(\epsilon)-\pi \Phi_{0}(\epsilon, \epsilon) f_{2}(\epsilon)-\int_{0}^{\infty}\left[\frac{\Phi_{+}(\epsilon, \omega)}{\omega-\epsilon}+\frac{\Phi_{-}(\epsilon, \omega)}{\omega+\epsilon}\right] f_{1}(\omega) d \omega=F_{1}(\epsilon)
$$

and

$$
\begin{aligned}
\pi \Phi_{0}(\epsilon, \epsilon) f_{1}(\epsilon)+ & \Lambda(\epsilon) f_{2}(\epsilon)-\int_{0}^{\infty}\left[\frac{\Phi_{+}(\epsilon, \omega)}{\omega-\epsilon}-\frac{\Phi_{-}(\epsilon, \omega)}{\omega+\epsilon}\right] f_{2}(\omega) d \omega \\
& -\int_{0}^{\infty} \frac{\Phi_{0}(\epsilon, \omega)}{\omega+\epsilon} f_{0}(\omega) d \omega=F_{2}(\epsilon)
\end{aligned}
$$

respectively.

\section{A SHORT-RANGE IMPURITY POTENTIAL MODEL}

In this section we solve the system of the integral equations derived above, see Eqs. (699) and (170), or the real equivalents of the latter - Eqs. (73) and (73), and then calculate the conductivity using Eq. (67) for a weak extremely short-range impurity potential.

\section{A. Formulation of the model}

Let us consider a zero-range impurity potential, which we define by $U(\mathbf{r})=U_{0} \Omega \delta(\mathbf{r})$, where the parameter $U_{0}$ has dimension of energy. For this potential, $U(\mathbf{q})=\Omega U_{0}$ so the kernels $\Phi_{\alpha}$ become independent of their energy arguments and all equal

$$
\Phi_{\alpha}(\epsilon, \omega)=\Phi=\frac{c U_{0}^{2} \Omega}{4 \pi v^{2} \hbar^{2}}
$$

Here where we have restored the Planck constant $\hbar$ to stress that $\Phi$ is dimensionless. This approximation poses no formal problem as regards the integral terms in Eqs. (69) and (70) but $\Lambda(\epsilon)$ acquires an ultraviolet logarithmic divergence. Thus some ultraviolet cut off 
procedure should be introduced. We define $\Phi_{\alpha}(\epsilon, \omega)=\Phi$ at $0 \leq \epsilon \leq \epsilon_{c}, 0 \leq \omega \leq \epsilon_{c}$ and zero otherwise. Then the simple calculation using Eqs. (71) and (64) yields

$$
\Lambda(\epsilon)=2-4 \Phi \int_{0}^{\epsilon_{c}} \frac{\omega}{\omega^{2}-\epsilon^{2}} d \omega=2\left[1-\Phi \ln \left(\frac{\epsilon^{2}}{\epsilon_{c}^{2}-\epsilon^{2}}\right)\right]
$$

at $\epsilon<\epsilon_{c}$ and $\Lambda(\epsilon)=2$ otherwise. This function has infinity breakpoint at $\epsilon=\epsilon_{c}$, which has no physical meaning. Analysis of a general case with a finite-range potential shows that the model is reasonable assuming that we are interested only in small enough energies in comparison with $\epsilon_{c}=\hbar v / r_{0}$ where $r_{0}$ is a characteristic radius of the potential. On the other hand, the noted mathematical property of the model $\Lambda(\epsilon)$ allows us to solve a part of the obtained singular integral equation exactly, see Appendix.

\section{B. The model integral equations and their solution}

Using the introduced model we obtain from the following system of singular integral equations for $\epsilon \leq \epsilon_{c}$

$$
\begin{gathered}
f_{2}(\epsilon)+f_{0}(\epsilon)-\frac{2}{\pi} \int_{0}^{\epsilon_{c}} \frac{f_{1}(\omega)}{\omega^{2}-\epsilon^{2}} \omega d \omega=0, \\
\Lambda(\epsilon) f_{1}(\epsilon)-\pi \Phi f_{2}(\epsilon)-2 \Phi \int_{0}^{\epsilon_{c}} \frac{f_{1}(\omega)}{\omega^{2}-\epsilon^{2}} \omega d \omega=F_{1}(\epsilon), \\
\pi \Phi f_{1}(\epsilon)+\Lambda(\epsilon) f_{2}(\epsilon)-2 \Phi \epsilon \int_{0}^{\epsilon_{c}} \frac{f_{2}(\omega)}{\omega^{2}-\epsilon^{2}} d \omega-\Phi \int_{0}^{\epsilon_{c}} \frac{f_{0}(\omega)}{\omega+\epsilon} d \omega=F_{2}(\epsilon),
\end{gathered}
$$

where

$$
F_{2}(\epsilon)=\frac{1}{2 \pi} \int_{0}^{\infty}\left[\frac{1}{\omega+\epsilon} \frac{\partial N_{0}(\omega)}{\partial \omega}-\frac{1}{\omega-\epsilon} \frac{\partial D_{0}(\omega)}{\partial \omega}\right] d \omega .
$$

At $\epsilon>\epsilon_{c}$ we have

$$
f_{1}(\epsilon)=\frac{1}{2} F_{1}(\epsilon) \approx-(4 \epsilon)^{-1}, F_{2}(\epsilon)=0
$$

so $f_{2}(\epsilon)=0$ in this range. Yet, no definite a priori information on $f_{0}(\epsilon)$ at $\epsilon>\epsilon_{c}$ can be deduced in the considered model.

This set of singular integral equations can be solved using the well-developed methods 37 of complex calculus, which is presented in Appendix. Using Eq. (118) from Appendix, we 
get for the function which directly determines the Zitterbewegung conductivity in Eq. (67)

$$
\begin{aligned}
f_{2}(\epsilon)-f_{0}(\epsilon) & =-\frac{2(\pi \Phi)^{2}}{(2 \pi \Phi)^{2}+\Lambda^{2}(\epsilon)} \frac{F_{1}(\epsilon)}{\pi \Phi}+\frac{\Lambda(\epsilon)}{\sqrt{\Lambda^{2}(\epsilon)+(2 \pi \Phi)^{2}}} \\
& \times \frac{2}{\pi} \int_{0}^{\epsilon_{c}} \frac{e^{\Theta\left(\epsilon^{2}\right)-\Theta\left(\omega^{2}\right)} F_{1}(\omega)}{\sqrt{\Lambda^{2}(\omega)+(2 \pi \Phi)^{2}}} \frac{\omega d \omega}{\omega^{2}-\epsilon^{2}}-\frac{2(\pi \Phi)^{2} f_{0}(\epsilon)}{(2 \pi \Phi)^{2}+\Lambda^{2}(\epsilon)} \\
& -\frac{2 \Phi \Lambda(\epsilon)}{\sqrt{\Lambda^{2}(\epsilon)+(2 \pi \Phi)^{2}}} \int_{0}^{\epsilon_{c}} \frac{e^{\Theta\left(\epsilon^{2}\right)-\Theta\left(\omega^{2}\right)} f_{0}(\omega)}{\sqrt{\Lambda^{2}(\omega)+(2 \pi \Phi)^{2}}} \frac{\omega d \omega}{\omega^{2}-\epsilon^{2}} .
\end{aligned}
$$

In turn, $f_{0}(\epsilon)$ satisfies Eq. (119) which is closed Fredholm like integral equation with a kernel non-singular at $\epsilon=\omega$. As we will see immediately below, the first term in Eq. (82) is compatible to a Zitterbewegung one obtained from qualitative analysis of the Kubo formula for ideal Dirac fermions. ${ }^{\underline{7}}$ The last two terms give rise a novel Zitterbewegung contribution to $\sigma$, which results in post-leading corrections, $O(1)$ at most, for $\Phi \rightarrow 0$ (see Appendix) and so neglected here. Note that, though local in the energy, the first term in Eq. (82) is just a result of solving the singular integral equations system, Eqs. (77) - (79), and not of plain approach when $f_{0}(\epsilon)$ and all the singular integral terms of the system are neglected in advance.

Using the adopted approximation in Eq.(156) yields for the Boltzmann conductivity part in the units $e^{2} / h$ the following 16

$$
\sigma_{\mathrm{B}}=-\frac{1}{2 \pi \Phi} \int_{0}^{\infty} \frac{\partial N_{0}(\epsilon)}{\partial \epsilon} d \epsilon=\frac{N_{0}(0)}{2 \pi \Phi}=\frac{1}{2 \pi \Phi},
$$

up to the terms $O\left(T \min \left(\mu^{-1}, \epsilon_{c}^{-1}\right)\right.$. Let us now integrate the first term in right-hand side of Eq. (82) over $\epsilon$ using integration by parts. Thus we obtain the Zitterbewegung contribution to $\sigma$ in a pseudo-Boltzmann form. Using Eq. (72), we have

$$
\begin{gathered}
\sigma_{\mathrm{ZB}}=\sigma_{\mathrm{B}} \int_{0}^{\epsilon_{c}} \frac{(\pi \Phi)^{2}}{(2 \pi \Phi)^{2}+\Lambda^{2}(\epsilon)}\left[\frac{1-N_{0}(\epsilon)}{\epsilon}-\frac{\partial D_{0}(\epsilon)}{\partial \epsilon}\right] d \epsilon \\
=-\int_{0}^{\infty}\left[\epsilon \tau_{\mathrm{ZB}}(\epsilon) \frac{\partial N_{0}(\epsilon)}{\partial \epsilon}-\epsilon \frac{\partial \epsilon \tau_{\mathrm{ZB}}(\epsilon)}{\partial \epsilon} \frac{\partial D_{0}(\epsilon)}{\partial \epsilon}\right] d \epsilon,
\end{gathered}
$$

where, by the definition,

$$
\tau_{\mathrm{ZB}}(\epsilon)=\frac{\sigma_{\mathrm{B}}}{\epsilon} \int_{\epsilon}^{\epsilon_{c}} \frac{(\pi \Phi)^{2}}{(2 \pi \Phi)^{2}+\Lambda^{2}(\omega)} \frac{d \omega}{\omega}
$$

is an effective Zitterbewegung relaxation time. 


\section{Applicability of classical Boltzmann equation and analysis of the $\mu=0$ case}

From Eq.(76) we find at $\epsilon \ll \epsilon_{c}$

$$
\Lambda(\epsilon) \approx 2\left(1+2 \Phi \ln \frac{\epsilon}{\epsilon_{c}}\right)
$$

i.e. $\Lambda(\epsilon)$ zeroes at the energy

$$
\epsilon_{\mathrm{K}}=\epsilon_{c} e^{-\frac{1}{2 \Phi}}=\epsilon_{c} e^{-\pi \sigma_{\mathrm{B}}}
$$

which is a striking analog of the Kondo energy scale in a problem of magnetic impurity in metals. ${ }^{38}$ Existence of this exponentially small energy scale in the problem under consideration was established first in Refs,11,12. Estimations of contributions from Eqs. (84) and Eqs. (85) as well the neglected Zitterbewegung terms show that at

$$
\epsilon_{c} \gg|\mu| \gg \max \left(\epsilon_{\mathrm{K}}, T\right)
$$

the corrections to the Bornian conductivity (83) are at most finite in the limit $\Phi \rightarrow 0$ and thus can be neglected in comparison with $\sigma_{\mathrm{B}}$. This justifies using the classical Boltzmann equation for graphene, except the case of extremally small doping.

Formally speaking, application of the theory developed here to the case of zero doping is doubtful. For example, the self-consistent Born approximation $\frac{16}{}$ gives for this case drastically different results in comparison with the Born approximation. At the same time, our approach is formally exact in a sense of perturbation theory at $\Phi \rightarrow 0$. We will see that, actually, the classical Born-approximation Boltzmann equation does not take into account properly all terms of order of $\Phi^{-1}$, a part of such results from Zitterbewegung.

Let us now perform integration in Eq. (85) assuming validity of Eq. (86), which is fairly justified at $\Phi \rightarrow 0$. This yields for the case $\mu=0$

$$
\epsilon \tau_{\mathrm{ZB}}(\epsilon)=\frac{\pi \sigma_{B}}{8}\left[\arctan \frac{1}{\Phi \pi}-\arctan \left(\frac{2}{\pi} \ln \frac{\epsilon}{\epsilon_{\mathrm{K}}}\right)\right] .
$$

Substituting Eq. (89) into Eq. (84), we obtain the integral formula for the conductivity in the undoped graphene $(\mu=0)$

$$
\sigma=\sigma_{B}\left\{1+\frac{\pi}{8} \int_{0}^{\infty}\left[\frac{\pi}{2}-\arctan \left(\frac{2}{\pi} \ln \frac{2 T}{\epsilon_{K}} x\right)\right] \frac{d x}{\cosh ^{2} x}\right\} .
$$

Given $\Phi \ll 1$, this formula has the following asymptotic behavior with respect to $T$

$$
\sigma \approx \sigma_{B}\left\{\begin{array}{c}
1+\frac{\pi^{2}}{4}+\frac{\pi^{2}}{16}\left(\ln \frac{\epsilon_{\mathrm{K}}}{T}\right)^{-1}, T \ll \epsilon_{\mathrm{K}} \\
1+\frac{\pi^{2}}{16}\left(\ln \frac{T}{\epsilon_{\mathrm{K}}}\right)^{-1}, T \gg \epsilon_{\mathrm{K}} .
\end{array}\right.
$$


It is seen that the Zitterbewegung correction at $T=0$ has the same order of magnitude as the Bornian conductivity and numerically even larger than it by a factor $\pi^{2} / 4$. The temperaturedependent corrections are reminiscent to those in the early theories of the Kondo effect by Abrikosov and Hamann. $\underline{38}^{2}$ Thus we obtain the following conductivity ratio

$$
\frac{\sigma(0)}{\sigma(\infty)}=1+\frac{\pi^{2}}{4}
$$

\section{CONCLUSIONS}

We have derived the second-order perturbational GKE for 2D massless Dirac fermions in graphene scattered by scalar impurity potential. We considered the GKE solution in the Dirac-delta potential model with the ultraviolet energy cutoff. Our principal result is the criterion given by Eq. (88), which justifies using the classical Boltzmann equation, except for exponentially narrow interval of chemical potential and temperature. Our approach clearly demonstrated that the problem of conductivity at zero doping it fairly similar to the Kondo problem. In this case, we obtained the temperature dependent conductivity formula, Eq. (90), which interpolates well between high-temperature $\left(T \gg \epsilon_{\mathrm{K}}\right)$ and low-temperature $\left(T \ll \epsilon_{\mathrm{K}}\right)$ ranges and remains finite at $T=\epsilon_{\mathrm{K}}$. Thus consistent asymptotic solving the integral equations that result from the derived GKE, we performed in this paper, proves equivalent to a partial summation of the perturbation terms $\propto \ln T$. Similar procedure was carried out for canonical Kondo model using the NSO method in Ref. 39.

By the noted analogy with the Kondo problem,, 38 Eq. (90) at $T>\epsilon_{\mathrm{K}}$ is asymptotically correct in the controllable small parameter $\Phi$, while we may not pretend to describe by it the low-temperature properties, in particular $\sigma(0)$ in detail. Nevertheless for all $T$, our kinetic equations by construction are more general than the Boltzmann one (even with scattering rate modified due to chirality of the current carriers). Therefore, the discrepancy by factor $\sim 3.5$ in the values of $\sigma(0)$ obtained, see Eq. (92), makes the Boltzmann equation probably not very good starting point for generalizations, such as the self-consistent Born approximation. $\underline{16}^{\underline{*}}$ Would the Kondo analogy goes pretty far, the observed ${ }^{\underline{1}} \sigma(0) \sim e^{2} / h$ might be an evidence that the system enters at $T \ll \epsilon_{\mathrm{K}}$ a non-perturbational strong effective coupling regime, where the conductivity attains so called unitary limit, $\underline{38}$ rather than the result of strong bare coupling $\Phi \gg 1$ adopted in Ref. 16 . 


\section{Acknowledgements}

This work was supported by the Stichting voor Fundamenteel Onderzoek der Materie (FOM), the Netherlands, and by the EuroMagNET.

\section{Appendix}

Here we present the solution of integral equations (77)-(79). Subtracting Eq.(177) from Eq.(78) we obtain purely algebraic linear equation

$$
\Lambda(\epsilon) f_{1}(\epsilon)-2 \pi \Phi f_{2}(\epsilon)-\pi \Phi f_{0}(\epsilon)=F_{1}(\epsilon)
$$

Let us exclude now $f_{1}(\epsilon)$ expressing it via $f_{0}(\epsilon)$ and $f_{2}(\epsilon)$. To this aim, we use Eq.(77) in the form of problem of inverting the Cauchy integral

$$
\frac{2}{\pi} \int_{0}^{\epsilon_{c}} \frac{f_{1}(\omega)}{\omega^{2}-\epsilon^{2}} \omega d \omega=f_{0}(\epsilon)+f_{2}(\epsilon) \equiv p_{1}(\epsilon) .
$$

In what follows we make use of the celebrated Poincare-Bertrand permutation formula 37 for the Cauchy-type integrals along a contour $C$ in complex plane

$$
\int_{C}\left[\frac{\psi(\epsilon, \tau)}{\tau-\epsilon} \int_{C} \frac{\varphi(\tau, \omega)}{\omega-\tau} d \omega\right] d \tau=\int_{C}\left[\int_{C} \frac{\psi(\epsilon, \tau) \varphi(\tau, \omega)}{\left(t_{1}-t\right)(\omega-\tau)} d \tau\right] d \omega-\pi^{2} \psi(\epsilon, \epsilon) \varphi(\epsilon, \epsilon) .
$$

Putting in Eq. (94) $\epsilon \rightarrow \tau, \frac{2 \omega}{\pi} \frac{f_{1}(\omega)}{\omega+\tau}=\varphi(\tau, \omega)$, multiplying it by

$$
\frac{\psi(\epsilon, \tau)}{\tau-\epsilon}=\frac{1}{\left(\tau^{2}-\epsilon^{2}\right) \sqrt{\epsilon_{c}^{2}-\tau^{2}}}
$$

and integrating over $\tau$ with the use Eq. (95), we obtain

$$
\begin{aligned}
\int_{0}^{\epsilon_{c}} \frac{p_{1}(\tau) d \tau}{\left(\tau^{2}-\epsilon^{2}\right) \sqrt{\epsilon_{c}^{2}-\tau^{2}}} & =-\frac{\pi f_{1}(\epsilon)}{2 \epsilon \sqrt{\epsilon_{c}^{2}-\epsilon^{2}}}+\frac{2}{\pi} \int_{0}^{\epsilon_{c}} \int_{0}^{\epsilon_{c}} \frac{1}{\left(\tau^{2}-\epsilon^{2}\right)\left(\omega^{2}-\tau^{2}\right)} \frac{d \tau}{\sqrt{\epsilon_{c}^{2}-\tau^{2}}} f_{1}(\omega) \omega d \omega \\
& =-\frac{\pi f_{1}(\epsilon)}{2 \epsilon \sqrt{\epsilon_{c}^{2}-\epsilon^{2}}}-\frac{2}{\pi} \int_{0}^{\epsilon_{c}} \frac{I\left(\omega^{2}\right)-I\left(\epsilon^{2}\right)}{\omega^{2}-\epsilon^{2}} \frac{d \tau}{\sqrt{\epsilon_{c}^{2}-\tau^{2}}} \frac{f_{1}(\omega)}{\omega^{2}-\epsilon^{2}} \omega d \omega,
\end{aligned}
$$

where (the integral below is the principal-value one)

$$
\begin{aligned}
I\left(\epsilon^{2}\right) & =\int_{0}^{\epsilon_{c}} \frac{d \tau}{\left(\tau^{2}-\epsilon^{2}\right) \sqrt{\epsilon_{c}^{2}-\tau^{2}}}=\frac{1}{\epsilon_{c}^{2}} \int_{0}^{\frac{\pi}{2}} \frac{d \chi}{\cos ^{2} \chi-\frac{\epsilon^{2}}{\epsilon_{c}^{2}}}=\frac{1}{\epsilon^{2}} \int_{0}^{\infty} \frac{d t}{\frac{\epsilon_{c}^{2}-\epsilon^{2}}{\epsilon^{2}}-t^{2}} \\
& =\frac{1}{\epsilon \sqrt{\epsilon_{c}^{2}-\epsilon^{2}}} \int_{0}^{\infty} \frac{d x}{1-x^{2}}=0
\end{aligned}
$$


from which we deduce that general solution is

$$
f_{1}(\epsilon)=-\frac{2 \epsilon \sqrt{\epsilon_{c}^{2}-\epsilon^{2}}}{\pi} \int_{0}^{\epsilon_{c}} \frac{p_{1}(\omega) d \omega}{\left(\omega^{2}-\epsilon^{2}\right) \sqrt{\epsilon_{c}^{2}-\omega^{2}}}+\frac{C_{1}}{\epsilon \sqrt{\epsilon_{c}^{2}-\epsilon^{2}}}
$$

provided that $p_{1}(\epsilon)$ is supposed to be known and $C_{1}$ is an arbitrary constant. Consider now the behavior of the above solution at the interval ends. We have identically

$$
\begin{aligned}
f_{1}(\epsilon) & =-\frac{2 \epsilon \sqrt{\epsilon_{c}^{2}-\epsilon^{2}}}{\pi} \int_{0}^{\frac{\pi}{2}} \frac{p_{1}\left(\epsilon_{c} \cos \alpha\right)}{\epsilon_{c} \cos ^{2} \alpha-\epsilon^{2}} d \alpha+\frac{C_{1}}{\epsilon \sqrt{\epsilon_{c}^{2}-\epsilon^{2}}} \\
& =-\frac{2 \epsilon \sqrt{\epsilon_{c}^{2}-\epsilon^{2}}}{\pi} \int_{0}^{\infty} p_{1}\left(\frac{\epsilon_{c}}{\sqrt{1+t^{2}}}\right) \frac{d t}{\epsilon_{c}^{2}-\epsilon^{2}-\epsilon^{2} t^{2}}+\frac{C_{1}}{\epsilon \sqrt{\epsilon_{c}^{2}-\epsilon^{2}}} \\
& =-\frac{2}{\pi} \int_{0}^{\infty} p_{1}\left(\frac{\epsilon_{c}}{\sqrt{1+\frac{\epsilon_{c}^{2}-\epsilon^{2}}{\epsilon^{2}} u^{2}}}\right) \frac{d u}{1-u^{2}}+\frac{C_{1}}{\epsilon \sqrt{\epsilon_{c}^{2}-\epsilon^{2}}}
\end{aligned}
$$

Thus, if $p_{1}(0)$ and $p_{1}\left(\epsilon_{c}\right)$ are finite, $f_{1}(\epsilon)$ at the ends diverges if $C_{1} \neq 0$ and is zero if $C_{1}=0$ since $\int_{0}^{\infty} \frac{d u}{1-u^{2}}=0$. Choosing $C_{1}=0$, we obtain from Eqs. (93) and (96) for the function

$$
q_{1}(\omega)=\frac{p_{1}(\omega)}{\omega \sqrt{\epsilon_{c}^{2}-\omega^{2}}}
$$

the following singular integral equation

$$
\begin{aligned}
\Lambda(\epsilon) \frac{2}{\pi} \int_{0}^{\epsilon_{c}} q_{1}(\omega) \frac{\omega d \omega}{\omega^{2}-\epsilon^{2}}+2 \pi \Phi q_{1}(\epsilon) & =\frac{\pi \Phi f_{0}(\epsilon)-F_{1}(\epsilon)}{\epsilon \sqrt{\epsilon_{c}^{2}-\epsilon^{2}}} \\
& \equiv p_{2}(\epsilon) .
\end{aligned}
$$

Introducing new variables and functions by

$$
\sqrt{x}=\epsilon, \sqrt{y}=\omega, \widehat{q}_{1}(x)=q_{1}(\sqrt{x}), \widehat{p}_{2}(x)=p_{2}(\sqrt{x})
$$

we convert Eq.(98) to the standard singular integral equation ${ }^{37}$

$$
\Lambda(\sqrt{x}) \frac{1}{\pi} \int_{0}^{x_{c}} \frac{\widehat{q}_{1}(y)}{y-x} d y+2 \pi \Phi \widehat{q}_{1}(x)=\widehat{p}_{2}(x), 0<x<x_{c}=\epsilon_{c}^{2},
$$

assuming $p_{2}(x)$ is known. Following the standard procedure ${ }^{37}$, we define the function of complex variable

$$
Q_{1}(z)=\frac{1}{2 \pi i} \int_{0}^{x_{c}} \frac{\widehat{q}_{1}(y)}{y-z} d y
$$

which is analytic in the plane with the cut along $\left(0, x_{c}\right)$ and

$$
\lim _{|z| \rightarrow \infty} Q_{1}(z)=0
$$


The relations at $z \rightarrow x \pm 0$

$$
\widehat{q}_{1}(x)=Q_{1}^{+}(x)-Q_{1}^{-}(x), \frac{1}{\pi i} \int_{0}^{x_{c}} \frac{\widehat{q}_{1}(y)}{y-x} d y=Q_{1}^{+}(x)+Q_{1}^{-}(x)
$$

map our equation onto the Riemann-Hilbert boundary value problem

$$
i \Lambda(\sqrt{x})\left[Q_{1}^{+}(x)+Q_{1}^{-}(x)\right]+2 \pi \Phi\left[Q_{1}^{+}(x)-Q_{1}^{-}(x)\right]=\widehat{p}_{2}(x),
$$

or

$$
Q_{1}^{+}(x)-G_{1}(x) Q_{1}^{-}(x)=\frac{\widehat{p}_{2}(x)}{2 \pi \Phi+i \Lambda(\sqrt{x})},
$$

where

$$
G_{1}(x)=\frac{2 \pi \Phi-i \Lambda(\sqrt{x})}{2 \pi \Phi+i \Lambda(\sqrt{x})} .
$$

Proceeding, we are to solve the homogeneous Riemann-Hilbert problem of searching a regular analytic function $\Omega_{1}(z)$ satisfying

$$
\Omega_{1}^{+}(x)=G_{1}(x) \Omega_{1}^{-}(x),
$$

which is considered below. To obtain the solution of the inhomogeneous problem following Ref.37 we divide Eq.(104) by $\Omega_{1}^{+}(x)$ and using Eq.(106) obtain

$$
\frac{Q_{1}^{+}(x)}{\Omega_{1}^{+}(x)}-\frac{Q_{1}^{-}(x)}{\Omega_{1}^{-}(x)}=\frac{1}{\Omega_{1}^{+}(x)} \frac{\widehat{p}_{2}(x)}{2 \pi \Phi+i \Lambda(\sqrt{x})},
$$

from which it immediately follows that

$$
Q_{1}(z)=\Omega_{1}(z)\left[\frac{1}{2 \pi i} \int_{0}^{x_{c}} \frac{1}{\Omega_{1}^{+}(y)} \frac{\widehat{p}_{2}(y)}{2 \pi \Phi+i \Lambda(\sqrt{y})} \frac{d y}{y-z}+P_{1}(z)\right],
$$

where $P_{1}(z)$ is an analytic function in the whole plane except may be points $z=0$ and $z=x_{c}$. The values of $Q_{1}(z)$ on real axis allows one to obtain $\widehat{q}_{1}(x)$ and its Cauchy integral using first of Eq.(103) along with Eq. (107) as follows

$$
\begin{aligned}
\widehat{q}_{1}(x) & =\left[\Omega_{1}^{+}(x)-\Omega_{1}^{-}(x)\right]\left[\frac{1}{2 \pi i} \int_{0}^{x_{c}} \frac{1}{\Omega_{1}^{+}(y)} \frac{\widehat{p}_{2}(y)}{2 \pi \Phi+i \Lambda(\sqrt{y})} \frac{d y}{y-x}+P_{1}(x)\right] \\
+ & \frac{1}{2}\left[1+\frac{\Omega_{1}^{-}(x)}{\Omega_{1}^{+}(x)}\right] \frac{\widehat{p}_{2}(x)}{2 \pi \Phi+i \Lambda(\sqrt{x})} \\
& =-\frac{\Lambda(\sqrt{x}) \Omega_{1}^{+}(x)}{2 \pi \Phi-i \Lambda(\sqrt{x})}\left[\frac{1}{\pi} \int_{0}^{x_{c}} \frac{1}{\Omega_{1}^{+}(y)} \frac{\widehat{p}_{2}(y)}{2 \pi \Phi+i \Lambda(\sqrt{y})} \frac{d y}{y-x}+2 i P_{1}(x)\right] \\
& +\frac{2 \pi \Phi \widehat{p}_{2}(x)}{(2 \pi \Phi)^{2}+\Lambda^{2}(\sqrt{x})}
\end{aligned}
$$


and

$$
\begin{aligned}
\frac{1}{\pi} \int_{0}^{x_{c}} \frac{\widehat{q}_{1}(y)}{y-x} d y & =\left[\Omega_{1}^{+}(x)+\Omega_{1}^{-}(x)\right]\left[\frac{1}{2 \pi} \int_{0}^{x_{c}} \frac{1}{\Omega_{1}^{+}(y)} \frac{\widehat{p}_{2}(y)}{2 \pi \Phi+i \Lambda(\sqrt{y})} \frac{d y}{y-x}+i P_{1}(x)\right] \\
& +\frac{i}{2}\left[1-\frac{\Omega_{1}^{-}(x)}{\Omega_{1}^{+}(x)}\right] \frac{\widehat{p}_{2}(x)}{2 \pi \Phi+i \Lambda(\sqrt{x})} \\
& =\frac{2 \pi \Phi \Omega_{1}^{+}(x)}{2 \pi \Phi-i \Lambda(\sqrt{x})}\left[\frac{1}{\pi} \int_{0}^{x_{c}} \frac{1}{\Omega_{1}^{+}(y)} \frac{\widehat{p}_{2}(y)}{2 \pi \Phi+i \Lambda(\sqrt{y})} \frac{d y}{y-x}+2 i P_{1}(x)\right] \\
+ & \frac{\Lambda(\sqrt{x}) \widehat{p}_{2}(x)}{(2 \pi \Phi)^{2}+\Lambda^{2}(\sqrt{x})} .
\end{aligned}
$$

Returning to the relevant homogeneous problem we assume that $\Omega_{1}(z) \neq 0, \infty$ at $z \neq 0$, $x_{c}$. Thus we arrive at the inhomogeneous problem for $\ln \Omega_{1}(z)$

$$
\ln \Omega_{1}^{+}(x)-\ln \Omega_{1}^{-}(x)=\ln G_{1}(x)=-2 i \arctan \frac{\Lambda(\sqrt{x})}{2 \pi \Phi} .
$$

Note that the end-point conditions are

$$
\lim _{x \rightarrow 0+} \ln G_{1}(x)=i \pi, \lim _{x \rightarrow x_{c}-0} \ln G_{1}(x)=-i \pi,
$$

where the first limit holds in general case and the second is the model property. Consider the following Cauchy integral ${ }^{37}$

$$
U_{1}(z)=\frac{1}{2 \pi i} \int_{0}^{x_{c}} \frac{\ln G_{1}(x)}{x-z} d x=-\frac{1}{\pi} \int_{0}^{x_{c}} \arctan \frac{\Lambda(\sqrt{x})}{2 \pi \Phi} \frac{d x}{x-z} .
$$

This function satisfies $\lim _{|z| \rightarrow 0} U_{1}(z)=0$. It has two regular nodes at the points $z=0$ and $z=x_{c}$, which is shown using integration by parts

$$
\begin{aligned}
U_{1}(z) & =-\left.\frac{1}{\pi} \ln (x-z) \arctan \frac{\Lambda(\sqrt{x})}{2 \pi \Phi}\right|_{0+} ^{x_{c}-0}+\frac{1}{\pi} \int_{0}^{x_{c}} \ln (x-z) \frac{d}{d x} \arctan \frac{\Lambda(\sqrt{x})}{2 \pi \Phi} d x \\
& =-\frac{1}{2} \ln \left[(-z)\left(x_{c}-z\right)\right]+2 \Phi \int_{0}^{x_{c}} \frac{\ln (x-z) \Lambda^{\prime}(\sqrt{x})}{\Lambda^{2}(\sqrt{x})+(2 \pi \Phi)^{2}} d x .
\end{aligned}
$$

Thus the function $e^{U_{1}(z)}$ can be taken for $\Omega_{1}(z)$, which satisfies $\lim _{|z| \rightarrow \infty} \Omega_{1}(z)=1$ and hence it should sustain $\lim _{|z| \rightarrow \infty} P_{1}(z)=0$. For this $\Omega_{1}(z)$ we have on real axis

$$
\begin{aligned}
\Omega_{1}^{ \pm}(x) & =e^{U_{1}(x \pm i 0)}=e^{-\frac{1}{\pi} \int_{0}^{x_{c}} \arctan \frac{\Lambda(\sqrt{y})}{2 \pi \Phi} \frac{d y}{y-x \mp i 0}}=e^{-\Theta(x) \mp i \arctan \frac{\Lambda(\sqrt{x})}{2 \pi \Phi}} \\
& =\frac{2 \pi \Phi \mp i \Lambda(\sqrt{x})}{\sqrt{\Lambda^{2}(\sqrt{x})+(2 \pi \Phi)^{2}}} e^{\Theta_{1}(x)}
\end{aligned}
$$


where

$$
\Theta_{1}(x)=-\frac{1}{\pi} \int_{0}^{x_{c}} \arctan \frac{\Lambda(\sqrt{y})}{2 \pi \Phi} \frac{d y}{y-x} .
$$

This yields with $P_{1}=0$

$$
\begin{aligned}
\widehat{q}_{1}(x) & =-\frac{\Lambda(\sqrt{x}) e^{\Theta_{1}(x)}}{\sqrt{\Lambda^{2}(\sqrt{x})+(2 \pi \Phi)^{2}}} \frac{1}{\pi} \int_{0}^{x_{c}} \frac{e^{-\Theta_{1}(y)} \widehat{p}_{2}(y)}{\sqrt{\Lambda^{2}(\sqrt{y})+(2 \pi \Phi)^{2}}} \frac{d y}{y-x} \\
& +\frac{2 \pi \Phi \widehat{p}_{2}(x)}{(2 \pi \Phi)^{2}+\Lambda^{2}(\sqrt{x})}
\end{aligned}
$$

and

$$
\begin{aligned}
\frac{1}{\pi} \int_{0}^{x_{c}} \frac{\widehat{q}_{1}(y)}{y-x} d y & =\frac{2 \pi \Phi e^{\Theta_{1}(x)}}{\sqrt{\Lambda^{2}(\sqrt{x})+(2 \pi \Phi)^{2}}} \frac{1}{\pi} \int_{0}^{x_{c}} \frac{e^{-\Theta_{1}(y)} \widehat{p}_{2}(y)}{\sqrt{\Lambda^{2}(\sqrt{y})+(2 \pi \Phi)^{2}}} \frac{d y}{y-x} \\
& +\frac{\Lambda(\sqrt{x}) \widehat{p}_{2}(x)}{(2 \pi \Phi)^{2}+\Lambda^{2}(\sqrt{x})} .
\end{aligned}
$$

Returning to the energy variables, we obtain

$$
p_{1}(\epsilon)=\frac{2(\pi \Phi)^{2} f_{3}(\epsilon)}{(2 \pi \Phi)^{2}+\Lambda^{2}(\epsilon)}-\frac{2 \Phi \Lambda(\epsilon)}{\sqrt{\Lambda^{2}(\epsilon)+(2 \pi \Phi)^{2}}} \int_{0}^{\epsilon_{c}} \frac{e^{\Theta\left(\epsilon^{2}\right)-\Theta\left(\omega^{2}\right)} f_{3}(\omega)}{\sqrt{\Lambda^{2}(\omega)+(2 \pi \Phi)^{2}}} \frac{\omega d \omega}{\omega^{2}-\epsilon^{2}}
$$

and

$$
f_{1}(\epsilon)=-\frac{\pi \Phi \Lambda(\epsilon) f_{3}(\epsilon)}{(2 \pi \Phi)^{2}+\Lambda^{2}(\epsilon)}-\frac{\pi(2 \Phi)^{2}}{\sqrt{\Lambda^{2}(\epsilon)+(2 \pi \Phi)^{2}}} \int_{0}^{\epsilon_{c}} \frac{e^{\Theta\left(\epsilon^{2}\right)-\Theta\left(\omega^{2}\right)} f_{3}(\omega)}{\sqrt{\Lambda^{2}(\omega)+(2 \pi \Phi)^{2}}} \frac{\omega d \omega}{\omega^{2}-\epsilon^{2}}
$$

where

$$
f_{3}(\epsilon)=f_{0}(\epsilon)-\frac{F_{1}(\epsilon)}{\pi \Phi}
$$

and

$$
\begin{aligned}
\Theta\left(\epsilon^{2}\right) & =\Theta_{1}\left(\epsilon^{2}\right)+\ln \sqrt{\epsilon^{2}\left(\epsilon_{c}^{2}-\omega^{2}\right)}=2 \Phi \int_{0}^{\epsilon_{c}} \frac{\Lambda^{\prime}(\sqrt{x})}{\Lambda^{2}(\sqrt{x})+(2 \pi \Phi)^{2}} \ln \left|x-\epsilon^{2}\right| d x \\
& =\Phi^{2} \epsilon_{c}^{2} \int_{0}^{\epsilon_{c}} \frac{d x}{\left(1+\Phi \ln \frac{x}{\epsilon_{c}^{2}-x}\right)^{2}+(\pi \Phi)^{2}} \frac{d \epsilon^{2} \mid}{x\left(\epsilon_{c}^{2}-x\right)} \\
& =\int_{-\infty}^{\infty} \ln \left|\frac{e^{u}}{e^{u}+1}-\frac{\epsilon^{2}}{\epsilon_{c}^{2}}\right| \frac{d u}{\left(\Phi^{-1}+u\right)^{2}+\pi^{2}}
\end{aligned}
$$

the constant $\Phi^{2} \ln \epsilon_{c}^{2}$ being omited since only difference $\Theta\left(\epsilon^{2}\right)-\Theta\left(\omega^{2}\right)$ enters all formulas. 
¿From Eq. (114) and the definition of $p_{1}(\epsilon)$ we obtain the connection between two functions of interest

$$
\begin{aligned}
f_{2}(\epsilon) & =-\frac{F_{1}(\epsilon)}{\pi \Phi}-\frac{2(\pi \Phi)^{2}+\Lambda^{2}(\epsilon)}{(2 \pi \Phi)^{2}+\Lambda^{2}(\epsilon)} f_{3}(\epsilon) \\
& -\frac{2 \Phi \Lambda(\epsilon)}{\sqrt{\Lambda^{2}(\epsilon)+(2 \pi \Phi)^{2}}} \int_{0}^{\epsilon_{c}} \frac{e^{\Theta\left(\epsilon^{2}\right)-\Theta\left(\omega^{2}\right) f_{3}(\omega)}}{\sqrt{\Lambda^{2}(\omega)+(2 \pi \Phi)^{2}}} \frac{\omega d \omega}{\omega^{2}-\epsilon^{2}} .
\end{aligned}
$$

Using Eqs.(115),(118) and (116) along with Eq.(79) and the Poincare-Bertrand formula, see Eq.(95), we obtain closed integral equation for the function $f_{0}(\epsilon)$. The equation reads

$$
\Lambda(\epsilon) f_{0}(\epsilon)-\Phi \int_{0}^{\epsilon_{c}}\left[Q(\epsilon, \omega)-\frac{1}{\omega+\epsilon}\right] f_{0}(\omega) d \omega=F_{3}(\epsilon),
$$

where the kernel and inhomogeneity term are given by

$$
\begin{aligned}
Q(\epsilon, \omega) & =\frac{1}{\omega^{2}-\epsilon^{2}}\left\{\frac{\omega\left[(2 \pi \Phi)^{2}+2 \Lambda^{2}(\epsilon)\right] e^{\Theta\left(\epsilon^{2}\right)-\Theta\left(\omega^{2}\right)}}{\sqrt{\Lambda^{2}(\epsilon)+(2 \pi \Phi)^{2}} \sqrt{\Lambda^{2}(\omega)+(2 \pi \Phi)^{2}}}\right. \\
& \left.-\frac{\epsilon\left[(2 \pi \Phi)^{2}+2 \Lambda^{2}(\omega)\right]}{(2 \pi \Phi)^{2}+\Lambda^{2}(\omega)}\right\}+\Phi K(\epsilon, \omega)
\end{aligned}
$$

and

$$
F_{3}(\epsilon)=-F_{2}(\epsilon)-\frac{1}{\pi} \int_{0}^{\epsilon_{c}}\left[\frac{2 \epsilon}{\omega^{2}-\epsilon^{2}}+Q(\epsilon, \omega)\right] F_{1}(\omega) d \omega
$$

respectively, while

$$
K(\epsilon, \omega)=\frac{4 \epsilon \omega}{\sqrt{\Lambda^{2}(\omega)+(2 \pi \Phi)^{2}}} \int_{0}^{\epsilon_{c}} \frac{e^{\Theta\left(\tau^{2}\right)-\Theta\left(\omega^{2}\right)} \Lambda(\tau) d \tau}{\left(\tau^{2}-\epsilon^{2}\right)\left(\omega^{2}-\tau^{2}\right) \sqrt{\Lambda^{2}(\tau)+(2 \pi \Phi)^{2}}}
$$

is another kernel. Note that, like $K(\epsilon, \omega)$, the kernel $Q(\epsilon, \omega)$ is non-singular at $\omega=\epsilon \neq 0$. Also we have

$$
\lim _{\Phi \rightarrow 0} K(\epsilon, \omega)=2 \epsilon \omega \int_{0}^{\epsilon_{c}} \frac{d \tau}{\left(\tau^{2}-\epsilon^{2}\right)\left(\omega^{2}-\tau^{2}\right)}=\frac{\omega \ln \left(\frac{\epsilon_{c}+\epsilon}{\epsilon_{c}-\epsilon}\right)-\epsilon \ln \left(\frac{\epsilon_{c}+\omega}{\epsilon_{c}-\omega}\right)}{\epsilon^{2}-\omega^{2}}
$$

and

$$
\lim _{\Phi \rightarrow 0} Q(\epsilon, \omega)=2 \frac{\omega-\epsilon}{\omega^{2}-\epsilon^{2}}=\frac{2}{\epsilon+\omega}=Q^{(0)}(\epsilon, \omega) .
$$

Further, the leading terms in $\Phi$ at $\Phi \ll 1$ of the inhomogeneity term is

$$
\lim _{\Phi \rightarrow 0} F_{3}(\epsilon)=-F_{2}(\epsilon)+\frac{2}{\pi} \int_{0}^{\epsilon_{c}} \frac{F_{1}(\omega) \omega}{\omega^{2}-\epsilon^{2}} d \omega=F_{3}^{(0)}(\epsilon) .
$$


It is clearly seen from the above equations that $f_{0}(\epsilon)=O(1)$ at $\Phi \rightarrow 0$, which results in the conductivity formula obtained in the main text.

1 A. K. Geim and K. S. Novoselov, Nature Mater. 6, 183 (2007).

2 M. I. Katsnelson, Mater. Today 10 (Issue 1-2), 20 (2007).

3 S. Das Sarma, A. K. Geim, P. Kim, and A. H. MacDonald (Editors), Special Issue of Solid State Commun. 143, 1-125 (2007).

4 G. W. Semenoff, Phys. Rev. Lett. 53, 2449 (1984).

5 F. D. M. Haldane, Phys. Rev. Lett. 61, 2015 (1988).

6 J. Gonzales, F. Guinea, and M. A. H. Vozmediano, Nucl. Phys. B 424, 595 (1994).

7 M. I. Katsnelson, Eur. Phys. J. B 51, 157 (2006).

8 M. I. Katsnelson, K. S. Novoselov, and A. K. Geim, Nature Phys. 2, 620 (2006).

9 V. V. Cheianov, V. I. Fal'ko, and B. L. Altshuler, Science 315, 1252 (2007).

10 M. I. Katsnelson and K. S. Novoselov, Solid State Commun. 143, 3 (2007).

11 E. Fradkin, Phys. Rev. B 33, 3263 (1986).

12 P. A. Lee, Phys. Rev. Lett. 71, 1887 (1993).

13 A. W. W. Ludwig, M. P. A. Fisher, R. Shankar, and G. Grinstein, Phys. Rev. B 50, 7526 (1994).

14 A. A. Nersesyan, A. M. Tsvelik, and F. Wenger, Phys. Rev. Lett. 72, 2628 (1994).

15 K. Ziegler, Phys. Rev. Lett. 80, 3113 (1998).

16 N. H. Shon and T. Ando, J. Phys. Soc. Japan 67, 2421 (1998).

17 R. Kubo, J. Phys. Soc. Japan 12, 570 (1957).

18 H. Nakano, Prog. Theor. Phys. 17, 147 (1957).

19 H. Mori, Prog. Theor. Phys. 34, 399 (1965).

20 J. M. Ziman, Electrons and Phonons - The Theory of Transport Phenomena in Solids (Oxford Univ. Press, Oxford, 2001).

21 K. Nomura and A. H. MacDonald, Phys. Rev. Lett. 96, 256602 (2006).

22 T. Ando, J. Phys. Soc. Japan 75, 074716 (2006).

23 E. W. Hwang, S. Adam, and S. DasSarma, Phys. Rev. Lett. 98, 186806 (2007).

24 M. I. Katsnelson and A. K. Geim, E-print at arXiv:0706.2490.

25 E. McCann, K. Kechedzhi, V. I. Falko, H. Suzuura, T. Ando, and B. L. Altshuler, Phys. Rev. 
Lett. 97, 146805 (2006).

26 P. M. Ostrovsky, I. V. Gornyi, and A. D. Mirlin, Phys. Rev. B 74, 235443 (2006).

27 I. L. Aleiner and K. B. Efetov, Phys. Rev. Lett. 97, 236801 (2006).

28 A. Altland, Phys. Rev. Lett. 97, 236802 (2006).

29 W. Kohn and J. M. Luttinger, Phys. Rev. 108, 590 (1957); J. M. Luttinger and W. Kohn, Phys. Rev. 109, 1892 (1958).

30 L. P. Kadanoff and G. Baym, Quantum Statistical Mechanics (W. A. Benjamin, Inc., New York, 1962).

31 L. V. Keldysh, Zh. Eksp. Teor. Fiz. 47, 1515 (1964) [Engl. transl.: Sov. Phys. - JETP 20, 1018 (1965)].

32 J. Rammer and H. Smith, Rev. Mod. Phys. 58, 323 (1984).

33 D. N. Zubarev, Nonequilibrium Statistical Thermodynamics (Consultants Bureau, New York, 1974).

34 R. Luzzi, A. R. Vasconcellos and J. G. Ramos, Int. J. Mod. Phys. B, 14, 3189 (2000).

35 A. L. Kuzemsky, arXiv: cond-mat/0502194 (2005).

36 A. I. Akhiezer and S. V. Peletminskii, Methods of Statistical Physics (Pergamon, Oxford, 1981).

37 N. I. Muskhelishvili, Singular Integral Equations (Dover, New York, 1992).

38 J. Kondo, Solid State Physics, vol.23 (Academic Press, New York, 1969), p.183.

39 V.P. Kalashnikov and M.I. Auslender, Fortschritte der Physik, 27, 355 (1979). 